\title{
Modulational instability in the nonlocal $\chi^{(2)}$-model
}

\author{
John Wyller ${ }^{\mathrm{a}, *}$, Wiesław Z. Królikowski ${ }^{\mathrm{b}}$, Ole Bang ${ }^{\mathrm{c}}$, Dan Erik Petersen ${ }^{\mathrm{d}}$, Jens Juul Rasmussen ${ }^{\mathrm{e}}$ \\ a Department of Mathematical Sciences and Technology, Norwegian University of Life Sciences, P.O. Box 5003, N-1432 Ås, Norway \\ ${ }^{\mathrm{b}}$ Laser Physics Centre, Research School of Physical Sciences and Engineering, Australian National University, Canberra, ACT 0200, Australia \\ ${ }^{\mathrm{c}}$ Research Center COM, Technical University of Denmark, DK-2800 Kgs. Lyngby, Denmark \\ ${ }^{\mathrm{d}}$ Department of Computer Science, University of Copenhagen, DK-2100 Kobenhavn Ø, Denmark \\ ${ }^{\mathrm{e}}$ Risф National Laboratory, Technical University of Denmark, Optics and Plasma Research Department, OPL-128, P.O. Box 49, DK-4000 Roskilde, Denmark
}

Received 12 May 2006; received in revised form 22 December 2006; accepted 2 January 2007

Available online 20 January 2007

Communicated by C.K.R.T. Jones

\begin{abstract}
We investigate in detail the linear regime of the modulational instability (MI) properties of the plane waves of the nonlocal model for $\chi^{(2)}$ media formulated in Nikolov et al. [N.I. Nikolov, D. Neshev, O. Bang, W.Z. Królikowski, Quadratic solitons as nonlocal solitons, Phys. Rev. E 68 (2003) 036614; I.V. Shadrivov, A.A. Zharov, Dynamics of optical spatial solitons near the interface between two quadratically nonlinear media, J. Opt. Soc. Amer. B 19 (2002) 596-602]. It is shown that the MI is of the oscillatory type and of finite bandwidth. Moreover, it is possible to identify regions in the parameter space for which a fundamental gain band exists, and regions for which higher order gain bands and modulational stability exist. We also show that the MI analysis for the nonlocal model is applicable in the finite walk-off case. Finally, we show that the plane waves of the nonlocal $\chi^{(2)}$-model are recovered as the asymptotic limit of one of the branches of the plane waves (i.e. the adiabatic branch or the acoustic branch) of the full $\chi^{(2)}$-model by means of a singular perturbational approach. It is also proven that the stability results for the adiabatic branch continuously approach those of the nonlocal $\chi^{(2)}$-model, by using the singular perturbational approach. The other branch of the plane waves (i.e. the nonadiabatic branch or the optical branch) is always modulationally unstable. We compare the MI results for the adiabatic branch with the predictions obtained from the full $\chi^{(2)}$-model in the non-walk-off limit. It is concluded that for most physical relevant parameter regimes it suffices to use the nonlocal model in order to determine the MI properties.
\end{abstract}

(C) 2007 Elsevier B.V. All rights reserved.

Keywords: Nonlocal systems; Nonlinear optics; Modulational instabilities; Singular perturbation theory

\section{Introduction}

Spatial nonlocality of the nonlinear response is a generic property of a wide range of physical systems, which manifests itself in new and exciting properties of nonlinear waves [1-6]. The nonlocality implies that the response of the medium at a given point depends not only on the wave function at that point (as in local media), but also on the wave function in its vicinity. The nonlocal nature often results from transport processes, such as atom diffusion [7], heat transfer [8,9] or drift of electric charges [10]. It can also be induced by a longrange molecular interaction as in nematic liquid crystals, which

\footnotetext{
* Corresponding author.

E-mail address: john.wyller@umb.no (J. Wyller).
}

exhibit orientational nonlocal nonlinearity [11,12]. Matter waves and Bose-Einstein condensates (BEC) inherently have a spatially nonlocal nonlinear response due to the finite range of the inter-particle interaction potential [13].

It turns out that the concept of nonlocality can be extended further to the nonlinear systems which are not nonlocal in the traditional sense as described above. These are systems involving interaction of few fields which while they rely on the local response of the medium can be modelled as being effectively spatially nonlocal. In particular, this is the case of the type I wave interaction process in quadratic media which has been shown to be equivalent (under certain conditions) to spatially nonlocal Kerr-type nonlinearity [14]. In order to show this equivalence, we will consider a fundamental wave (FW) and its second harmonic (SH) propagating along the $z$-direction in a lossless quadratic nonlinear medium under the conditions 
for type I phase matching. The normalized dynamical equations for the slowly varying envelopes $E_{1,2}(x, z)$ are then given as $[15,17]$

$$
\begin{aligned}
& \mathrm{i} \partial_{z} E_{1}+d_{1} \partial_{x}^{2} E_{1}+E_{1}^{*} E_{2} \exp (-\mathrm{i} \beta z)=0, \\
& \mathrm{i} \partial_{z} E_{2}+d_{2} \partial_{x}^{2} E_{2}+E_{1}^{2} \exp (\mathrm{i} \beta z)=0 .
\end{aligned}
$$

In the spatial domain $d_{1} \approx 2 d_{2}, d_{j}>0$, and the coordinate $x$ represents a transverse spatial direction. The term $\partial_{x}^{2} E_{j}$ then models the beam diffraction. In the temporal domain $d_{j}$ models the group velocity dispersion (GVD-) coefficient, which may take arbitrary values and $x$ represents time. In that case $\partial_{x}^{2} E_{j}$ accounts for the pulse dispersion. The parameter $\beta$ is the normalized phase-mismatch and $j=1,2$.

The modulational stability of plane/continuous waves described by Eqs. (1) and (2) was addressed in [16], where different regimes of modulational instability were found. A thorough survey of the nonlinear propagation of waves in $\chi^{(2)}$ media with respect to modulational instability/continuous waves, existence and stability of solitary waves is given in [17].

We conveniently rewrite the system (1) and (2) in the form

$$
\begin{aligned}
& \mathrm{i} \partial_{z} e_{1}+d_{1} \partial_{x}^{2} e_{1}+e_{1}^{*} e_{2}=0 \\
& \mathrm{i} \partial_{z} e_{2}-\beta e_{2}+d_{2} \partial_{x}^{2} e_{2}+e_{1}^{2}=0
\end{aligned}
$$

by means of the transformation

$$
E_{1}=e_{1}, \quad E_{2}=e_{2} \exp (\mathrm{i} \beta z) .
$$

A simplified version of the model (3) and (4) can be derived in the cascading limit, in which we assume a slow variation of $e_{2}(x, z)$ with respect to both $x$ and $z$ as compared to the phase mismatch variation, i.e., $\left|\partial_{z} e_{2}\right| \sim\left|d_{2} \partial_{x}^{2} e_{2}\right| \ll\left|\beta e_{2}\right|$, which means that the terms $\partial_{z} e_{2}, d_{2} \partial_{x}^{2} e_{2}$ are discarded in (3) and (4). In that case Eq. (4) for $\mathrm{SH}$ is approximated with

$e_{2}=e_{1}^{2} / \beta=E_{1}^{2} / \beta$

and, hence, Eq. (1) for the FW can be simplified to the conventional Nonlnear Schrödinger (NLS-) equation [15]

$\mathrm{i} \partial_{z} E_{1}+d_{1} \partial_{x}^{2} E_{1}+\beta\left|E_{1}\right|^{2} E_{1}=0$.

In [18] the $\chi^{(2)}$-model (1) and (2) has been investigated for large but finite mismatch parameter $\beta$. They derived a relation between the FW and the $\mathrm{SH}$ amplitudes by expanding in inverse powers of $\beta$, thus ending up with a nonlinear Schrödinger equation supplemented with terms of $O\left(\beta^{-2}\right)$ accounting for the group velocity mismatch (GVM) and the difference between the group velocity dispersion (GVD) of the FF and the SH.

The sign of the mismatch $\beta$ in (6) determines whether the effective Kerr nonlinearity is focusing or defocusing, and thus the cascading limit predicts modulational stability and dark solitons (MI and bright solitons) provided $\beta d_{1}<0\left(\beta d_{1}>0\right)$. However, it is well known that the cascading limit (6) yields predictions with respect to certain dynamical features which contradict those obtained from the full model. For example, the model (6) yields modulational stability of dark quadratic solitons for all values of $d_{2}$, whereas this is known to depend on the value of $d_{2}$ for the full model. Moreover, it predicts that the bright solitons are unstable for higher spatial dimensions and that they will either spread out or collapse, whereas it is known that stable quadratic solitons exist in all spatial dimensions and that collapse cannot occur in the full $\chi^{(2)}$-system [17,19-21].

Hence there is a need for a more accurate model which captures typical dynamical features of the $\chi^{(2)}$-model (1) and (2) and which at the same time is accessible for a simple physical interpretation. A step in that direction was proposed by Nikolov et al. [14]. Following that paper we only assume a slow variation of $e_{2}(x, z)$ in the propagation direction, i.e., that

$\left|\partial_{z} e_{2}\right| \ll\left|d_{2} \partial_{x}^{2} e_{2}\right| \sim\left|\beta e_{2}\right|$.

Thus, neglecting only $\mathrm{i} \partial_{z} e_{2}$ in (4), we solve (4) exactly using Fourier transformation and the convolution theorem, treating $E_{1}^{2}$ as a source term. In this case we can express $E_{2}$ as [14]

$E_{2}=\beta^{-1} N \exp [\mathrm{i} \beta z]$

$N\left(E_{1}^{2}\right)(x)=\left(R * E_{1}^{2}\right)(x) \equiv \int_{-\infty}^{\infty} R(x-y) E_{1}^{2}(y) \mathrm{d} y$,

with the response kernel $R(x)$ defining the nonlocality given as

$R(x)=\frac{1}{\sigma} \Phi_{s_{2}}(\xi), \quad \xi=\frac{x}{\sigma}$

where

$\Phi_{s_{2}}(\xi)= \begin{cases}\frac{1}{2} \exp (-|\xi|) & \text { if } s_{2}=+1 \\ \frac{1}{2} \sin (|\xi|) & \text { if } s_{2}=-1 .\end{cases}$

Here

$s_{2}=\operatorname{sign}\left(d_{2} \beta\right), \quad \sigma=\left|\frac{d_{2}}{\beta}\right|^{1 / 2}$.

Hence, when assuming slow variation of the second harmonic amplitude $e_{2}$ with respect to the propagation coordinate, i.e., (7), the system (1) and (2) can formally be approximated by the nonlocal equation [14]

$\mathrm{i} \partial_{z} E_{1}+d_{1} \partial_{x}^{2} E_{1}+\beta^{-1} N\left(E_{1}^{2}\right) E_{1}^{*}=0$,

with $N\left(E_{1}^{2}\right)$ given as $(8 \mathrm{~b})$.

Notice the difference between the nonlocal model (8)-(12) for the $\chi^{(2)}$ system and the generic Kerr type nonlocal model treated in [1-6]: In the $\chi^{(2)}$ model the convolution integral involves $E_{1}^{2}$, while in the Kerr type it involves the intensity $\left|E_{1}\right|^{2}$, thus some phase information is retained in (8)-(12).

Due to the form of the response function $R(x)$, the case $s_{2}=$ +1 is referred to as the nonresonant case or the exponentially decaying case, while the $s_{2}=-1$ is called the resonant case or oscillatory case. The Fourier transform of the response kernel $R(x)$ is given as

$\widetilde{R}(k)=\frac{1}{1+s_{2} \sigma^{2} k^{2}}$ 
from which it follows that $\widetilde{R}$ has real singularities given as

$k= \pm \frac{1}{\sigma}= \pm\left|\frac{\beta}{d_{2}}\right|^{1 / 2}$

when $s_{2}=-1$. In this case the response function (9) and (10) is derived by means of the inversion theorem for Fouriertransforms in the principal value sense and that this function cannot be normalized. The role of the real singularities (14) becomes even more transparent when studying the Fouriertransformed version of the SH-equation (4):

$\mathrm{i} \partial_{z} \widetilde{e}_{2}-\beta \widetilde{e}_{2}-d_{2} k^{2} \widetilde{e}_{2}+\widetilde{e}_{1}^{2}=0$.

Here $\widetilde{e}_{2}$ and $\widetilde{e_{1}^{2}}$ denote the Fourier transforms of $e_{2}$ and $e_{1}^{2}$, respectively. The evolution equation (15) clearly displays that $-\beta \widetilde{e}_{2}-d_{2} k^{2} \widetilde{e}_{2}$ will be comparable with the small term $\mathrm{i} \partial_{z} \widetilde{e}_{2}$ in the vicinity of the singularities (14) in the case $s_{2}=-1$. This naturally leads to the conjecture that the solutions of the initial value problem of (8)-(12) does not mimic the dynamical evolution of the full system (1) and (2) for this case.

A similar type of behavior occurs in the multidimensional analogue of (1) and (2), which recently has been used to model light bullets and $X$-waves in quadratic media [22]. When neglecting the slowly varying term $i \partial_{z} e_{2}$ by assuming (7), one ends up with a multidimensional analogue to (8)-(12). It was shown that the local cascading limit in terms of the nonlinear Schrödinger equation does not exist in the normal SH case, which reduces to the case $s_{2}=-1$ in the $1 \mathrm{D}$ case. By using the same type of arguments as in the 1D case, one might expect that the nonlocal approximation does not provide an appropriate description in the normal SH case as a dynamical model, since there is a wave number regime in the vicinity of the singularities of the spectrum of the response function for which the omission of the term $i \partial_{z} e_{2}$ is not justified [22].

In spite of these problems the model (8)-(12) represents an improved model of quadratic material as compared with the cascading limit (6). In [14] the Eqs. (8)-(12) which resemble the nonlocal Kerr media was used to model solitons in the full $\chi^{(2)}$-system. Moreover, the properties of the quadratic solitons are shown to exhibit a sensitive dependence on the parameter $d_{2}$, and the tendency to collapse is shown to be arrested in the nonlocal model (8)-(12).

Notice also that when taking into account walk-off effects, the full $\chi^{(2)}$-model (1) and (2) is extended to [15,17]

$\mathrm{i} \partial_{z} E_{1}+\mathrm{i} r_{1} \partial_{x} E_{1}+d_{1} \partial_{x}^{2} E_{1}+E_{1}^{*} E_{2} \exp (-\mathrm{i} \beta z)=0$,

$\mathrm{i} \partial_{z} E_{2}+\mathrm{i} r_{2} \partial_{x} E_{2}+d_{2} \partial_{x}^{2} E_{2}+E_{1}^{2} \exp (\mathrm{i} \beta z)=0$.

Here the parameters $r_{j}, j=1,2$ account for the walk-off effect in the fundamental wave equation $(j=1)$ and the second harmonic wave equation $(j=2)$. Notice that the walk-off term can be removed from the second harmonic equation (17) by changing to a coordinate frame moving with the velocity $r_{2}$, i.e., $x \rightarrow x-r_{2} t$, which produces the term $\mathrm{i}\left(r_{1}-r_{2}\right) \partial_{x} E_{1}$ in the first harmonic equation (16) accounting for the difference between the second harmonic and first harmonic walk-off. Hence we may without loss of generality put $r_{2}=0$ in (16) and interpret $r_{1}$ as the difference between the first harmonic and second harmonic walk-off coefficient. Now, by introducing the transformation (5) and making the slowness assumption (7), we can express the second harmonic field $E_{2}$ in terms of the first harmonic field, i.e., $E_{2}=\beta^{-1} N\left(E_{1}^{2}\right) \exp (\mathrm{i} \beta z)$, $N\left(E_{1}^{2}\right)(x)=\int_{-\infty}^{\infty} R(x-y) E_{1}^{2}(y) \mathrm{d} y$ with the response kernel given as (9), just as in the non-walk-off case treated in the introduction. The governing equation for the first harmonic field assumes the form (8)-(12) when referring the wave motion to a coordinate system moving with the speed $r_{1}$. Hence the inclusion of walk-off effects has no influence on wave phenomena like modulational instability, existence of stationary soliton solutions and noncollapse, when assuming slow variation of the second harmonic field with respect to the propagation coordinate $z$.

This background serves as a motivation for the present study. Our main goal is to link the dynamical properties of the nonlocal system consisting of (8) and (12) with those of the full $\chi^{(2)}$-model (1) and (2). Here we focus on the MI properties of the two models. The reason for this is that MI is a very important effect appearing only in nonlinear systems, which is even more fundamental than solitons, in that MI acts as a precursor to solitons.

We start out with investigating MI of plane waves within the framework of the nonlocal model (8)-(12). In this case it turns out that the gain spectrum depends on the two parameters $\gamma \equiv \frac{\rho_{0} \sigma^{4}}{\left|d_{1} d_{2}\right|}$ and $\eta_{0} \equiv \sigma^{2} k_{0}^{2}$ in addition to the two sign parameters $s_{1} \equiv \operatorname{sign}\left(\beta d_{1}\right)$ and $s_{2} \equiv \operatorname{sign}\left(\beta d_{2}\right)$. Here $\rho_{0}$ and $k_{0}$ denote the intensity and the wave number of the plane background wave. The parameter $\gamma$ is termed the effective degree of nonlocality. The key point in this analysis is to describe the MI in terms of a structural function in the four different cases $s_{1}= \pm 1, s_{2}= \pm 1$ in a way analogous to [3] for the MI in the conventional nonlocal NLS model. The structural function in the present case is a rational function of $\eta=$ $\sigma^{2} k^{2}$, where $k$ is the modulational wave number of the wave perturbation imposed on the plane wave. The coefficients of this function depend on the parameters $\left(\eta_{0}, \gamma\right)$. The numerator of this function contains a quintic polynomial, i.e., a product of a quadratic polynomial and a cubic polynomial, and in analogy with the procedure developed in [3] the MI analysis consists of determining the number of strictly positive zeros of this polynomial as a function of the parameters $\left(\eta_{0}, \gamma\right)$. It turns out that the number of such zeros determines the structure of the MI, i.e., the number of gain bands. The analysis shows that the maximum number of gain bands is three, one fundamental gain band and two higher order bands. Notice here that there is no fixed notation for what we call higher-order gain bands. They are also referred to as "secondary gain bands". Moreover, we have chosen to refer to the instability under consideration as MI for both the fundamental gain band and the higher order gain bands, following the terminology used in for example [23].

Secondly, we will compare the formal MI results obtained within the framework of the nonlocal model with the predictions deduced from the cascading limit (6) as well as the full $\chi^{(2)}$-model (1) and (2). The comparison between the full $\chi^{(2)}$-model and the corresponding nonlocal model is carried 
out by means of a singular perturbational approach. Notice that the latter approach has been elaborated in detail in the context of finite dimensional dynamical systems. Adapting to the terminology used for such systems, the nonlocal model can be said to play the role of the outer approximation or the adiabatic approximation of the solution to the full $\chi^{(2)}$-model (1) and (2). For a more extensive review of singular perturbation methods, we refer to Wasow [24] and Vasil'eva et al. [25].

The present paper is organized in the following way: In Section 2 the plane wave solution of the nonlocal model (12) is derived, and the dispersion relation for the modulations imposed on the plane wave background is derived, using (12) as a starting point. In Section 3 we introduce the concept of structural function and describe the general framework for determining the gain band structure by means of the positive zeros of the structural function. In Section 4 we apply this formalism to elaborate the details of the problem of MI when the response function is given as an exponentially decaying function, i.e., $s_{2}=+1$. The same problem is addressed for the oscillatory response function, i.e., $s_{2}=-1$ in Section 5. In Section 6 we first derive the two branches of the plane waves of the full $\chi^{(2)}$-model (1) and (2) and detect the branch which continuously deforms to the plane wave solution of (12) by using a singular perturbational approach. Secondly, the MI problem of both plane wave branches of the perturbed model (1) and (2) is investigated by means of the singular perturbational approach. Section 7 contains the conclusions and an outlook.

\section{Plane waves and MI in the nonlocal $\chi^{(2)}$-model}

We insert the plane wave ansatz

$E_{1}=A_{0} \exp \left[\mathrm{i} \theta_{0}\right], \quad \theta_{0}=k_{0} x-\omega_{0} z$

in the nonlocal model (8)-(12). Notice that the property of gauge invariance implies that we without loss of generality may assume that $A_{0}>0$. We derive the nonlinear, nonlocal dispersion relation

$\omega_{0}=d_{1} k_{0}^{2}-\beta^{-1} \rho_{0} \widetilde{R}\left(2 k_{0}\right), \quad \rho_{0} \equiv A_{0}^{2}$.

Here $\widetilde{R}$ denotes the Fourier transform of the response function (13). Thus the plane wave of the nonlocal model (8)-(12) depends on the spectrum $\widetilde{R}\left(2 k_{0}\right)$ of the response function, which is completely different from the spectrum independent plane wave of the nonlocal Kerr model [1-6]. This is indeed a consequence of the phase dependent nonlocal nonlinearity of (8)-(12).

Let us consider the plane wave (18) and (19) in the case $s_{2}=-1$ within the framework of Eq. (4) under the approximation (7), i.e., when neglecting the term $\mathrm{i} \partial_{z} e_{2}$. In that case (4) becomes equivalent to a linear driven oscillator equation, with a pumping frequency equal to $2 k_{0}$. When this frequency matches the natural frequency $1 / \sigma$ of (4), we have a linear resonance, which means that the second harmonic $e_{2}$ contains unphysical secular terms of the type $x \exp (\mathrm{i} x / \sigma)$ and $x \exp (-\mathrm{i} x / \sigma)$. The background wave number $k_{0}$ producing the resonance is given by the singularity of the Fourier transform of the response function $R(x)$ in the case $s_{2}=-1$ :

$k_{0}=k_{0}^{\mathrm{res}} \equiv \pm \frac{1}{2}\left|\frac{\beta}{d_{2}}\right|^{1 / 2}$.

Hence the nonlocal model (12) is not applicable for plane waves in the vicinity of the resonance point (20), consistent with the general remark in the introduction about the validity of the nonlocal approximation.

Next, we perform a formal stability analysis of the plane waves (18)-(19) within the framework of the nonlocal approximation (8)-(12): by making the assumption

$E_{1}=\left[A_{0}+A_{1}(x, z)\right] \exp \left[\mathrm{i} \theta_{0}\right]$

where $A_{1}$ is a complex valued function. By linearizing around $A_{0} \gg\left|A_{1}\right|$, we obtain the evolution equation

$$
\begin{aligned}
& \mathrm{i} \partial_{z} A_{1}+d_{1} \partial_{x}^{2} A_{1}+2 \mathrm{i} d_{1} k_{0} \partial_{x} A_{1} \\
& \quad-2 \mathrm{i} \beta^{-1} \rho_{0} \widetilde{R}\left(2 k_{0}\right) \operatorname{Im}\left(A_{1}\right)+2 \beta^{-1} \rho_{0} R_{2 k_{0}} * A_{1}=0
\end{aligned}
$$

for the perturbation $A_{1}$. Here $R_{2 k_{0}} * A_{1}$ denotes the convolution integral

$\left(R_{2 k_{0}} * A_{1}\right)(x) \equiv \int_{-\infty}^{\infty} R(y-x) \exp \left[2 \mathrm{i} k_{0}(y-x)\right] A_{1}(y) \mathrm{d} y$.

The next step consists of referring the wave motion to a coordinate system moving with the group velocity, i.e.,

$\omega_{0}^{\operatorname{lin}}=d_{1} k_{0}^{2}, \quad v_{g}=\frac{\mathrm{d} \omega_{0}^{\operatorname{lin}}}{\mathrm{d} k_{0}}=2 d_{1} k_{0}$,
$\tau=z, \quad \xi=x-v_{g} z, \quad a_{1}(\xi, \tau)=A_{1}(x, z)$

from which the linearized nonlocal evolution equation for the wave perturbation $a_{1}$ becomes

$$
\begin{aligned}
& \mathrm{i} \partial_{\tau} a_{1}+d_{1} \partial_{\xi}^{2} a_{1}-2 \mathrm{i} \beta^{-1} \rho_{0} \widetilde{R}\left(2 k_{0}\right) \operatorname{Im}\left(a_{1}\right) \\
& \quad+2 \beta^{-1} \rho_{0} R_{2 k_{0}} * a_{1}=0 .
\end{aligned}
$$

We then decompose the perturbation $a_{1}$ into a real and an imaginary part, $a_{1}=u_{1}+\mathrm{i} v_{1}$, and obtain from (25) the coupled system of equations

$$
\begin{aligned}
& \partial_{\tau} u_{1}+d_{1} \partial_{\xi}^{2} v_{1}-2 \beta^{-1} \rho_{0} \widetilde{R}\left(2 k_{0}\right) v_{1}+2 \beta^{-1} \rho_{0}\left(I_{3}+I_{4}\right)=0 \\
& \partial_{\tau} v_{1}-d_{1} \partial_{\xi}^{2} u_{1}-2 \beta^{-1} \rho_{0}\left(I_{1}-I_{2}\right)=0 .
\end{aligned}
$$

Here $I_{i}, i=1,2,3,4$ denote the convolution integrals

$$
\begin{aligned}
& I_{1}=\int_{-\infty}^{\infty} R(y-\xi) \cos \left[2 k_{0}(y-\xi)\right] u_{1}(y) \mathrm{d} y \\
& I_{2}=\int_{-\infty}^{\infty} R(y-\xi) \sin \left[2 k_{0}(y-\xi)\right] v_{1}(y) \mathrm{d} y \\
& I_{3}=\int_{-\infty}^{\infty} R(y-\xi) \sin \left[2 k_{0}(y-\xi)\right] u_{1}(y) \mathrm{d} y \\
& I_{4}=\int_{-\infty}^{\infty} R(y-\xi) \cos \left[2 k_{0}(y-\xi)\right] v_{1}(y) \mathrm{d} y .
\end{aligned}
$$


The final step consists in converting the system (26) and (27) to a coupled set of ordinary differential equations by means of the Fourier-transformation technique. In accordance with that, we introduce

$\widetilde{u}_{1}(k) \equiv \int_{-\infty}^{\infty} u_{1}(\xi) \exp [\mathrm{i} k \xi] \mathrm{d} \xi$

$\widetilde{v}_{1}(k) \equiv \int_{-\infty}^{\infty} v_{1}(\xi) \exp [\mathrm{i} k \xi] \mathrm{d} \xi$.

Then, by exploiting the convolution theorem for Fourier transforms, we deduce the system

$$
\begin{aligned}
& \partial_{\tau} \widetilde{u}_{1}-d_{1} k^{2} \widetilde{v}_{1}-2 \beta^{-1} \rho_{0} \widetilde{R}\left(2 k_{0}\right) \widetilde{v}_{1} \\
& \quad+\beta^{-1} \rho_{0}\left[-\mathrm{i} \widetilde{\mu}_{-} \widetilde{u}_{1}+\widetilde{\mu}_{+} \widetilde{v}_{1}\right]=0 \\
& \partial_{\tau} \widetilde{v}_{1}+d_{1} k^{2} \widetilde{u}_{1}-\beta^{-1} \rho_{0}\left[\widetilde{\mu}_{+} \widetilde{u}_{1}+\mathrm{i} \widetilde{\mu}_{-} \widetilde{v}_{1}\right]=0 .
\end{aligned}
$$

Here we have introduced the shifts $\widetilde{\mu}_{ \pm}$of the Fourier transform $\widetilde{R}$ defined as

$$
\begin{aligned}
& \widetilde{\mu}_{+}\left(k, 2 k_{0}\right) \equiv \widetilde{R}\left(2 k_{0}+k\right)+\widetilde{R}\left(2 k_{0}-k\right) \\
& \widetilde{\mu}_{-}\left(k, 2 k_{0}\right) \equiv \widetilde{R}\left(2 k_{0}+k\right)-\widetilde{R}\left(2 k_{0}-k\right) .
\end{aligned}
$$

Then, by introducing the phase transformation

$\underline{X}=\exp \left(\mathrm{i} \beta^{-1} \rho_{0} \tilde{\mu}_{-} \tau\right) \underline{Y}, \quad \underline{X}=\left[\widetilde{u}_{1}, \widetilde{v}_{1}\right]^{T}$

the system (29) and (30) can be rewritten in compact matrix form as

$$
\partial_{\tau} \underline{Y}=\mathbf{A} \cdot \underline{Y}
$$

where $\mathbf{A}$ is defined as

$\mathbf{A}=\left(\begin{array}{cc}0 & 2 \beta^{-1} \rho_{0} \widetilde{R}\left(2 k_{0}\right)+d_{1} k^{2}-\beta^{-1} \rho_{0} \tilde{\mu}_{+} \\ \beta^{-1} \rho_{0} \tilde{\mu}_{+}-d_{1} k^{2} & 0\end{array}\right)$.

The stability problem is investigated by means of the eigenvalues $\lambda$ of the matrix $\mathbf{A}$. The characteristic equation of this matrix is given by

$$
\begin{aligned}
\lambda^{2}= & -\operatorname{det}(\mathbf{A})=\left(\beta^{-1} \rho_{0} \tilde{\mu}_{+}-d_{1} k^{2}-2 \beta^{-1} \rho_{0} \widetilde{R}\left(2 k_{0}\right)\right) \\
& \times\left(\beta^{-1} \rho_{0} \tilde{\mu}_{+}-d_{1} k^{2}\right)
\end{aligned}
$$

and it constitutes the basis for our study of MI within the framework of the nonlocal model (8)-(12).

Since the Fourier transform $\widetilde{R}$ is an even function of its argument, the shift $\widetilde{\mu}_{+}$is also an even function of the argument $k$. Therefore the coefficient matrix $\mathbf{A}$ is a function of $\eta \equiv k^{2}$. The stability problem can be studied solely in terms of the $\eta$-dependent invariants of the matrix $\mathbf{A}$, i.e., the trace and the determinant of $\mathbf{A}$. Then, following the stability methodology described in [26] (which is a generalized version of the Turing instability problem for reaction diffusion equations), the present stability investigation can be translated into a study of the behavior of the parameterized curve $\underline{C}$

$\underline{C}(\eta)=(\operatorname{tr}(\mathbf{A})(\eta), \operatorname{det}(\mathbf{A})(\eta))=(0, \operatorname{det}(\mathbf{A})(\eta)), \quad \eta \geq 0$

in the invariant plane of the coefficient matrix $\mathbf{A}$. If $\operatorname{det}(\mathbf{A})(\eta)>$ 0 for all $\eta \geq 0$, we have stability while the situation
$\operatorname{det}(\mathbf{A})(\eta)<0$ for some $\eta$ interval yields instability. The gain band structure can be elaborated in detail by means of the behavior of the curve $\underline{C}$. Notice that the same type of problem was encountered in the study presented in [3] regarding MI in nonlocal Kerr media. In the next section we will describe the present MI problem in detail.

\section{The structural function for the nonlocal problem and modulational stability}

In this section we rewrite the eigenvalue equation (34) in a form which is better suited for analytical treatment. To this end, we scale the wave numbers $k$ and $k_{0}$ against the reference value $1 / \sigma=\sqrt{\left|\beta / d_{2}\right|}$, and the eigenvalue $\lambda$ against the reference value $\lambda_{0} \equiv d_{1} \sigma^{-2}$, i.e.,

$\bar{k}_{0}=\sigma k_{0}, \quad \bar{k}=\sigma k, \quad \Lambda=\frac{\lambda}{\lambda_{0}}$

and introduce the effective degree of nonlocality $\gamma$ defined as

$\gamma=\frac{\rho_{0} \sigma^{4}}{\left|d_{1} d_{2}\right|}$

and the dimensionless spectrum $\widetilde{\Phi}\left(\bar{k} ; s_{2}\right)$ defined as

$\widetilde{\Phi}\left(\bar{k} ; s_{2}\right)=\frac{1}{1+s_{2} \bar{k}^{2}}$

with the corresponding shifts

$\tilde{\mu}_{+}\left(k, 2 k_{0}, s_{2}\right)=\widetilde{\Phi}\left(2 \bar{k}_{0}+\bar{k}, s_{2}\right)+\widetilde{\Phi}\left(2 \bar{k}_{0}-\bar{k}, s_{2}\right)$.

Moreover, let us introduce the structural function $\Psi$ defined by

$$
\begin{aligned}
& \Psi\left(\bar{k} ; 2 \bar{k}_{0}, s_{1}, s_{2}, \gamma\right) \\
& \quad=\left[s_{1} \gamma \tilde{\mu}_{+}-\bar{k}^{2}\right]\left[2 s_{1} \gamma \tilde{\mu}_{0}-s_{1} \gamma \tilde{\mu}_{+}+\bar{k}^{2}\right]
\end{aligned}
$$

where

$\widetilde{\mu}_{0} \equiv \widetilde{\Phi}\left(2 \bar{k}_{0}, s_{2}\right)=\frac{1}{1+4 s_{2} \bar{k}_{0}^{2}}$

and $\widetilde{\mu}_{+}$is the shift given as (38). Then the dispersion relation (34) can be rewritten in the form

$\Lambda^{2}=\Psi\left(\bar{k} ; 2 \bar{k}_{0}, \gamma, s_{1}, s_{2}\right)$.

Let us first consider some special cases:

(1) The linear regime. The special case $\gamma=0$ indeed reproduces the well known result of modulational stability of linear waves. Notice that this result holds true irrespective of the values of the normalized wave number $\bar{k}_{0}$ of the background plane wave.

(2) The cascading limit. By putting $\sigma=0$ in (13) one recovers the cascading limit, i.e., $\hat{\Phi}(\bar{k}) \equiv 1$. In that case we get

$\tilde{\mu}_{+}=2$

from which

$\Lambda^{2}=\left[2 s_{1} \gamma-\bar{k}^{2}\right] \bar{k}^{2}$ 
follows. Then by employing the scalings (35), one recovers the dispersion relation for the modulations of the cascading limit. When $\beta d_{1}<0$, then $s_{1}=-1$, and from the dispersion relation we get modulational stability. In the complementary regime, i.e., for $\beta d_{1}>0\left(\Leftrightarrow s_{1}=+1\right)$ one recovers the well known classical Lighthill MI result [27].

Let us now return to the general case. The MI problem can now be rephrased in the following manner:

(1) If for given input parameters $s_{1}, s_{2}, \gamma$ and $\bar{k}_{0}$, we have $\Psi\left(\bar{k} ; 2 \bar{k}_{0}, \gamma, s_{1}, s_{2}\right)<0$ for all $\bar{k}$, then the plane wave (18) and (19) is modulationally stable.

(2) If for given input parameters $s_{1}, s_{2}, \gamma$ and $\bar{k}_{0}$ there is a range of $\bar{k}$-values for which the structural function $\Psi\left(\bar{k} ; 2 \bar{k}_{0}, \gamma, s_{1}, s_{2}\right) \geq 0$, then the plane wave (18) and (19) is modulationally unstable. In this case the growth rate $\Gamma$ of the instability can be expressed as

$\Gamma \equiv|\operatorname{Re}(\Lambda)|=\sqrt{\Psi\left(\bar{k} ; 2 \bar{k}_{0}, \gamma, s_{1}, s_{2}\right)}$.

(3) Since $\widetilde{\Phi} \rightarrow 0$ as $|\bar{k}| \rightarrow \infty$, we have $\Psi\left(\bar{k} ; 2 \bar{k}_{0}, \gamma, s_{1}, s_{2}\right) \rightarrow$ $-\bar{k}^{4}$ as $|\bar{k}| \rightarrow \infty$. Hence any MI is always of the finite bandwidth type.

(4) The MI is always of an oscillatory type for a finite nonzero background wave number $\bar{k}_{0}$ due to the presence of the phase factor in the transformation (31).

Notice that the structural function satisfies the symmetry relations

$\Psi\left(\bar{k} ; 2 \bar{k}_{0}\right)=\Psi\left(-\bar{k} ;-2 \bar{k}_{0}\right), \quad \Psi\left(\bar{k} ; 2 \bar{k}_{0}\right)=\Psi\left(-\bar{k} ; 2 \bar{k}_{0}\right)$,

$\Psi\left(\bar{k} ; 2 \bar{k}_{0}\right)=\Psi\left(\bar{k} ;-2 \bar{k}_{0}\right)$

which easily are inferred from the fact that the Fourier transform $\widetilde{\Phi}$ is an even function of its arguments.

The above symmetry properties of the structural function show that without loss of generality we can study the MIproblem for $\bar{k} \geq 0$ and $\bar{k}_{0} \geq 0$. In the next sections we will detail the MI problem with respect to existence of fundamental gain bands, higher order gain bands and possibility of having modulational stability. In both the cases $s_{2}=+1$ and $s_{2}=-1$ it turns out that the structural function $\Psi$ is a rational function which can be written in the form

$$
\begin{aligned}
\Psi & \left(\bar{k} ; 2 \bar{k}_{0}, \gamma, s_{1}, s_{2}\right) \\
& =\frac{\eta P_{3}\left(\eta ; \eta_{0}, \gamma, s_{1}, s_{2}\right) Q_{2}\left(\eta ; \eta_{0}, \gamma, s_{1}, s_{2}\right)}{\left(F_{2}\left(\eta ; \eta_{0}, s_{2}\right)\right)^{2}} \\
\eta & =\bar{k}^{2} \quad \eta_{0}=\bar{k}_{0}^{2}
\end{aligned}
$$

where $P_{3}, Q_{2}$ and $F_{2}$ are polynomials

$$
\begin{aligned}
& P_{3}\left(\eta ; \eta_{0}, \gamma, s_{1}, s_{2}\right)=-\eta^{3}+p_{2} \eta^{2}+p_{1} \eta+p_{0}, \\
& Q_{2}\left(\eta ; \eta_{0}, \gamma, s_{1}, s_{2}\right)=\eta^{2}+q_{1} \eta+q_{0}, \\
& F_{2}\left(\eta ; \eta_{0}, s_{2}\right)=\eta^{2}+f_{1} \eta+f_{0} .
\end{aligned}
$$

The expressions for coefficients of these polynomials are presented in the Appendix.
The zeros of $F_{2}$ (=singularities of the structural function $\Psi$ ) are given as

$\eta_{ \pm}=4 \eta_{0}-s_{2} \pm 4 \sqrt{-s_{2} \eta_{0}}$

and they are complex in the case $s_{2}=+1$ and real for $s_{2}=-1$. Assume that we are in the regimes producing real singularities of the structural function $\Psi$, i.e., when $s_{2}=-1$. We now prove that these singularities are of order 2 and belong to an $\eta$-interval for which the structural function is negative. This means that the presence of these singularities does not affect the formation of the gain band structure. The proof proceeds as follows: First we observe that $\eta_{ \pm}>0$ and that $\eta_{+} \neq \eta_{-}$provided $\eta_{0}>0$. Then we find that

$\left[\eta P_{3} Q_{2}\right]_{\eta=\eta_{ \pm}}=-64 \eta_{ \pm} \gamma^{2} \eta_{0}<0$

which shows that $\Psi \rightarrow-\infty$ as $\eta \rightarrow \eta_{ \pm}$. A separate investigation for $\eta_{0}=0$ reveals that even in this case the zeros $\eta_{ \pm}$define singularities of order 2 of the structural function, simply by observing that $\eta=1$ is a double zero of $P_{3} Q_{2}$ for $\eta_{0}=0$ and that

$\left[\eta P_{3} Q_{2}\right]_{\eta=1}=-4 \gamma^{2}<0$

and thus the structural function goes to $-\infty$ as $\eta$ approaches the singularity $\eta=1$. In the following we will often refer to the set $\Delta$ defined as

$\Delta=\left\{\left(\eta_{0}, \gamma\right) \mid \eta_{0} \geq 0, \gamma \geq 0\right\}$

as the control set and the elements in this set as control vectors. Notice that for the case $s_{2}=-1$ one also has to exclude the singular point $\eta_{0}=\frac{1}{4}$ from the control space $\Delta$, since this particular point corresponds to the resonance point (20). We refer to this line in the control set as the resonance line.

The product $H_{5} \equiv P_{3} Q_{2}$ is a quintic polynomial of the type

$$
\begin{aligned}
& H_{5}\left(\eta ; \eta_{0}, \gamma, s_{1}, s_{2}\right)=P_{3}\left(\eta ; \eta_{0}, \gamma, s_{1}, s_{2}\right) Q_{2}\left(\eta ; \eta_{0}, \gamma, s_{1}, s_{2}\right) \\
& \quad=-\eta^{5}+b_{4} \eta^{4}+b_{3} \eta^{3}+b_{2} \eta^{2}+b_{1} \eta+b_{0}
\end{aligned}
$$

where all the coefficients are real and depend on the control vector $\left(\eta_{0}, \gamma\right)$ and the two sign parameters $s_{1}$ and $s_{2}$. By exploiting the expressions for the polynomials $P_{3}$ and $Q_{2}$ as given in the Appendix, we find that $b_{0}=p_{0} q_{0}$ is given as

$b_{0}=-2 s_{1} \gamma\left[2\left(12 s_{1} \eta_{0}-s_{1} s_{2}\right) \gamma-s_{2}\left(4 \eta_{0}+s_{2}\right)^{3}\right]$

while the expressions for the remaining coefficients $b_{1}, b_{2}, b_{3}$ and $b_{4}$ are quite lengthy and complicated and therefore will not be reproduced here. Notice that the sign of the structural function $\Psi$ is equal to the sign of the quintic polynomial $\mathrm{H}_{5}$, so that the MI problem formulated previously translates into a study of $\mathrm{H}_{5}$. i.e.,

Now, by taking the asymptotic behavior of $H_{5}$ into account,

$H_{5}\left(\eta ; \eta_{0}, \gamma, s_{1}, s_{2}\right) \sim-\eta^{5} \quad$ as $\eta \rightarrow \infty$

we are now in the position to formulate a classification scheme for the stability problem, which essentially relies on the properties of the zeros of the polynomial $\mathrm{H}_{5}$ : 
Table 1

The relation between the gain band structure and the number of strictly positive zeros of $\mathrm{H}_{5}$ in the transversal crossing case

\begin{tabular}{ll}
\hline Number of positive zeros of $\mathrm{H}_{5}$ & Gain band structure \\
\hline 0 & Modulational stability \\
1 & Fundamental gain band \\
2 & One higher order gain band \\
3 & Fundamental gain band \\
& + one higher order gain band \\
5 & Two higher order gain bands \\
& Fundamental gain band \\
& + two higher order gain bands \\
\hline
\end{tabular}

(1) Sufficient condition for MI. Existence of fundamental gain bands. Let the sign parameters $s_{1}$ and $s_{2}$ be given. If $H_{5}(\eta=0)=b_{0}>0$ for a given control vector $\left(\eta_{0}, \gamma\right)$, then the quintic polynomial $H_{5}$ has at least one positive real zero. By continuity, $H_{5}\left(\eta ; \eta_{0}, \gamma, s_{1}, s_{2}\right)>0$ for some $\eta$-interval about 0 , which means that the structural function $\Psi$ is positive in that particular interval, from which it follows that we always have MI in that case. Since according to the fundamental theorem of algebra a quintic polynomial has five zeros (counted with multiplicity), there are a maximum of four additional positive zeros of $H_{5}$. Moreover, since the coefficients of $H_{5}$ are real the polynomial can in total either have one, three or five positive real zeros, corresponding to the existence a fundamental gain band only, a fundamental gain band+one higher order gain band or a fundamental gain band+two higher order gain bands, respectively. To determine the exact number of higher order gain bands as a function of the control set $\Delta$, one has to study the number of positive zeros of the polynomials $P_{3}$ and $Q_{2}$ in detail. To conclude, the condition $b_{0}>0$ is a sufficient condition for having MI.

(2) Necessary condition for modulational stability. Assume that $s_{1}$ and $s_{2}$ are given. If $H_{5}(\eta=0)=b_{0}<0$ for a given control vector $\left(\eta_{0}, \gamma\right)$, then the quintic polynomial $H_{5}$ has at least one negative real zero. In this case the fundamental theorem of algebra implies that there are a maximum of four positive real zeros of $\mathrm{H}_{5}$, and since the coefficients of $H$ are real, we can either have no, two or four positive real zeros in total. The first case corresponds to modulational stability, the second one to one higher order gain band while the last situation to two higher order gain bands. Also in this case the positive zeros of the polynomials $P_{3}$ and $Q_{2}$ are important when determining the number of gain bands. To summarize, if we have modulational stability, then $b_{0}<0$. In this case we have no fundamental gain band.

Table 1 summarizes the gain band structure as a function of the number of positive zeros of the quintic polynomial $H_{5}$ for a given set of sign parameters $\left(s_{1}, s_{2}\right)=( \pm 1, \pm 1)$.

The number of positive zeros of the quintic polynomial $\mathrm{H}_{5}$ as a function of the control space $\Delta$ is computed by means of the counting principle. The control set is naturally divided into a union of open disjoint subsets $\Delta_{i}^{f}, f=P_{3}, Q_{2}$ where the number of positive zeros of $P_{3}$ and $Q_{2}$ is constant on $\Delta_{i}^{P_{3}}$ and $\Delta_{i}^{Q_{2}}$, respectively. The boundaries of these subsets are given by the nontransversal crossings $f(\eta)=0=$ $f^{\prime}(\eta)=0, f=P_{3}, Q_{2}$. Then on any nonempty intersection $\Delta_{i}^{Q_{2}} \cap \Delta_{j}^{P_{3}}$ the number of positive zeros of $H_{5}$ is given as the sum of the zeros of $P_{3}$ and $Q_{2}$. A general formulation of this principle is presented in Appendix. An important feature in the analysis is detection of curves in the control-space for which the nontransversality condition is fulfilled. We treat these two type of conditions separately:

(1) Existence and nonexistence of fundamental gain bands. The condition $b_{0}=0$ is by (48) equivalent to

$$
\gamma=\phi\left(\eta_{0} ; s_{1}, s_{2}\right) \equiv \frac{s_{2}\left(4 \eta_{0}+s_{2}\right)^{3}}{2\left(12 s_{1} \eta_{0}-s_{1} s_{2}\right)} \text {. }
$$

The function $\phi$ describes a separatrix curve in the control set $\Delta$ for a given set of sign parameters $s_{1}$ and $s_{2}$. This curve separates regions in the control set for which fundamental gain bands exist from regions with nonexistence of such bands. Hence a fundamental gain band will either vanish or be converted to a higher order gain band when crossing this curve.

(2) Formation and coalescence of gain bands through a bifurcation process. Due to the factorization $\mathrm{H}_{5}=$ $Q_{2} P_{3}$ a nontransversal crossing of $H_{5}$ takes place if the transversality condition is not satisfied anymore for one of the polynomials $Q_{2}$ and $P_{3}$, i.e.,

$$
\begin{aligned}
& f\left(\eta ; \eta_{0}, \gamma, s_{1}, s_{2}\right)=f^{\prime}\left(\eta ; \eta_{0}, \gamma, s_{1}, s_{2}\right)=0, \\
& \quad f=Q_{2}, P_{3}
\end{aligned}
$$

(50) determines specific curves (separatrices) in the control set for a given set of sign parameters $s_{1}$ and $s_{2}$. The situation described by means of (50) corresponds to coalescence of two real zeros and formation of complex zeros when passing through points on the curves in the control space given by (50). When the real part of the zeros is positive, there are two possibilities for the gain band structure: Either a higher order gain band vanishes or two gain bands merge together. Notice that the gain spectrum of the instability in the latter situation behaves like $\sim\left|\bar{k}-\bar{k}_{\mathrm{cr}}\right|$ in the vicinity of the double zero $\eta_{\mathrm{cr}} \equiv \bar{k}_{\mathrm{cr}}^{2}$, i.e., it displays a cusp type of behavior.

For the quadratic polynomial $Q_{2}$ the process of vanishing and coalescence of gain bands can be described in a simple analytical way by means of the discriminant $D_{2}$ of $Q_{2}$. Simple computation reveals that

$$
\begin{aligned}
& D_{2}\left(\eta_{0}, \gamma, s_{1}, s_{2}\right)=q_{1}^{2}-4 q_{0} \\
& =4 \frac{\left(\gamma-\psi_{+}\left(\eta_{0}, s_{1}, s_{2}\right)\right)\left(\gamma-\psi_{-}\left(\eta_{0}, s_{1}, s_{2}\right)\right)}{\left(4 \eta_{0}+s_{2}\right)^{2}}
\end{aligned}
$$

where

$$
\begin{aligned}
\psi_{ \pm}\left(\eta_{0}, s_{1}, s_{2}\right)= & 4\left(4 \eta_{0}+s_{2}\right)\left(-2 s_{1} s_{2} \eta_{0}\right. \\
& \left. \pm\left(\eta_{0}\left(4 \eta_{0}+s_{2}\right)\right)^{1 / 2}\right) .
\end{aligned}
$$

In the case of the cubic polynomial $P_{3}$, an analysis of the monotonicity properties of $P_{3}$ will show for which parameter regimes the nontransversality condition (50) can be fulfilled. One computes the derivative of $P_{3}$ and 
finds that the discriminant $D_{3}$ of the resulting quadratic polynomial is given by $D_{3}=p_{2}^{2}+3 p_{1}$. For regimes where $D_{3} \leq 0$, (50) cannot be satisfied. In the complementary regime a careful analysis reveals that (50) only possesses solutions in the following regimes:

- $P_{3}(\eta=0)=p_{0}>0, p_{1}<0$ and $p_{2}>0$.

- $P_{3}(\eta=0)=p_{0}<0$ and $p_{1}>0$.

- $P_{3}(\eta=0)=p_{0}<0, p_{1}<0$ and $p_{2}>0$.

Thus we can conclude by appealing to (A.3) and (A.4) that there are only certain subsets of the control set $\Delta$ for which the nontransversality condition (50) holds for a given set of sign parameters $\left(s_{1}, s_{2}\right)$. Notice that the condition $D_{3}=0$ is equivalent to the curve $\gamma=\theta\left(\eta_{0}\right)$ in the control set where

$\theta\left(\eta_{0}\right)=-\frac{1}{6} s_{1} s_{2}\left(16 \eta_{0}^{2}-56 s_{2} \eta_{0}+1\right)$.

In the subsequent analysis we will make use of the conditions $p_{1}=0, p_{2}=0$ and $p_{3}=0$ whenever they are fulfilled as well as the signs of $P_{3}\left(z_{-}\right)$and $P_{3}\left(z_{+}\right)$. The condition $p_{1}=0$ is represented by the function $\gamma=\zeta\left(\eta_{0}\right)$ defined as

$\zeta\left(\eta_{0}\right)=\frac{1}{2} s_{1} s_{2}\left(4 \eta_{0}+s_{2}\right)^{2}, \quad s_{1}=s_{2}$.

In the subsequent two sections we demonstrate some aspects of the gain band structure as a function of the four sign parameters $\left(s_{1}, s_{2}\right)=( \pm 1, \pm 1)$ by using the methodological framework above. Here the main focus is put on the following aspects:

- Identification of parameter regimes producing modulational stability and MI.

- Conversion of a fundamental gain band to a higher order gain bands.

- Regimes producing only one fundamental gain band.

We also show the coalescence and splitting of gain bands and the existence of three gain bands without pursuing the mathematical details. The theoretical predicted gain band curves are compared with the gain spectra deduced from numerical simulations of the nonlocal model (8)-(12). The simulations are based on a Fourier split-step code in which the time dependence is resolved by means of the fourth order Runge-Kutta method.

\section{Modulational instability for the exponentially decaying response function $\left(s_{2}=+1\right)$}

\subsection{The gain band structure in the case $s_{1}=+1, s_{2}=+1$}

The description of the gain band structure is based on the properties of the polynomials $H_{5}, Q_{2}$ and $P_{3}$ as shown in the previous section. Since $P_{3}(\eta=0)=2 \gamma\left(4 \eta_{0}+1\right)>0$, it turns out that the quintic polynomial $H_{5}$ possesses at least one strictly positive zero originating from the polynomial $P_{3}$, and hence according to the classification scheme described in the previous section the plane waves always are modulationally unstable for $s_{1}=+1, s_{2}=+1$.
Moreover one can easily describe the regimes for which $Q_{2}$ possesses zero, one and two strictly positive zeros in terms of the separatrix functions $\phi$ and $\psi$ defined by (49) and (52) which in this case read

$\gamma=\phi\left(\eta_{0}\right)=\frac{\left(4 \eta_{0}+1\right)^{3}}{24 \eta_{0}-2}, \quad \eta_{0}>\frac{1}{12}$,
$\psi_{+}\left(\eta_{0}\right)=4\left(4 \eta_{0}+1\right)\left(-2 \eta_{0}+\left(\eta_{0}\left(4 \eta_{0}+1\right)\right)^{1 / 2}\right)$.

Finally, one can show that the bifurcation condition $P_{3}(\eta)=$ $P_{3}^{\prime}(\eta)=0$ can only take place in the regime $\eta_{0}>\frac{1}{4}, \theta\left(\eta_{0}\right)<$ $\gamma<\zeta\left(\eta_{0}\right)$ where

$\theta\left(\eta_{0}\right)=-\frac{8}{3} \eta_{0}^{2}+\frac{28}{3} \eta_{0}-\frac{1}{6}$,

$\zeta\left(\eta_{0}\right)=\frac{1}{2}\left(4 \eta_{0}+1\right)^{2}$.

We are now in a position to identify regimes producing fundamental gain bands. For $0 \leq \eta_{0} \leq \frac{1}{12}, \gamma>0$ and $\eta_{0}>$ $\frac{1}{12}, \gamma<\phi\left(\eta_{0}\right)$ the gain band structure contains a fundamental band, while for $\gamma$ exceeding the threshold $\phi\left(\eta_{0}\right)$ this type of gain bands ceases to exist.

In the process of detailing the gain band structure even further as a function of the input parameters $\left(\eta_{0}, \gamma\right)$, i.e., identifying as well as splitting and merging of gain bands one has to use the methodological framework elaborated in the previous section. We do not pursue all the details here, but rather point out some important features which we have illustrated by means of plots of gain spectra.

One can, for example, show that the fundamental gain band existing for $0 \leq \eta_{0} \leq \frac{1}{4}+\sqrt{5} \frac{1}{10}, \gamma<\phi\left(\eta_{0}\right)$ is always converted to a higher order gain band as we pass the curve $\gamma=$ $\phi\left(\eta_{0}\right)$. This is due to the fact that the number of strictly positive zeros of $Q_{2}$ always increases by one as we pass this curve for $0 \leq \eta_{0} \leq \frac{1}{4}+\sqrt{5} \frac{1}{10}$, while it vanishes for $\eta_{0}>\frac{1}{4}+\sqrt{5} \frac{1}{10}$ when crossing the same curve since in that case the number of positive zeros of $Q_{2}$ decreases by one. If $0 \leq \eta_{0} \leq \frac{1}{4}, \gamma<\phi\left(\eta_{0}\right)$, a careful analysis reveals that $P_{3}$ has only one strictly positive zero while $Q_{2}$ has none, and hence the gain band structure only consists of the fundamental gain band in that regime. Moreover, we can conclude that for $\gamma>\max \left(\phi\left(\eta_{0}\right), \zeta\left(\eta_{0}\right)\right)$, the exact number of strictly positive zeros of $H_{5}$ is 2 , and hence, we have proven the existence of only one higher order gain band in this case.

Finally, the process of splitting of gain bands can be predicted as $\gamma$ exceeds the threshold $\gamma=\psi_{+}\left(\eta_{0}\right)$ as well as when the bifurcation condition $P_{3}\left(\eta ; \eta_{0}, \gamma\right)=P_{3}^{\prime}\left(\eta ; \eta_{0}, \gamma\right)=$ 0 is fulfilled. Notice also that it is only in the regime $\eta_{0}>\frac{1}{4}$, $\theta\left(\eta_{0}\right)<\gamma<\zeta\left(\eta_{0}\right)$ the latter bifurcation condition is fulfilled and where we can have coexistence of a fundamental and two higher order gain bands.

In Fig. 1 semi-logarithmic plots of the control space with the curves (55)-(58) and $P_{3}\left(\eta ; \eta_{0}, \gamma\right)=P_{3}^{\prime}\left(\eta ; \eta_{0}, \gamma\right)=0$ are displayed. Based on Fig. 1 we select parameters $\left(\eta_{0}, \gamma\right)$ for which the analytical framework predicts conversion of fundamental gain bands to higher order gain band (Fig. 2(a)), conversion of a fundamental gain band to a higher order gain 
(a)

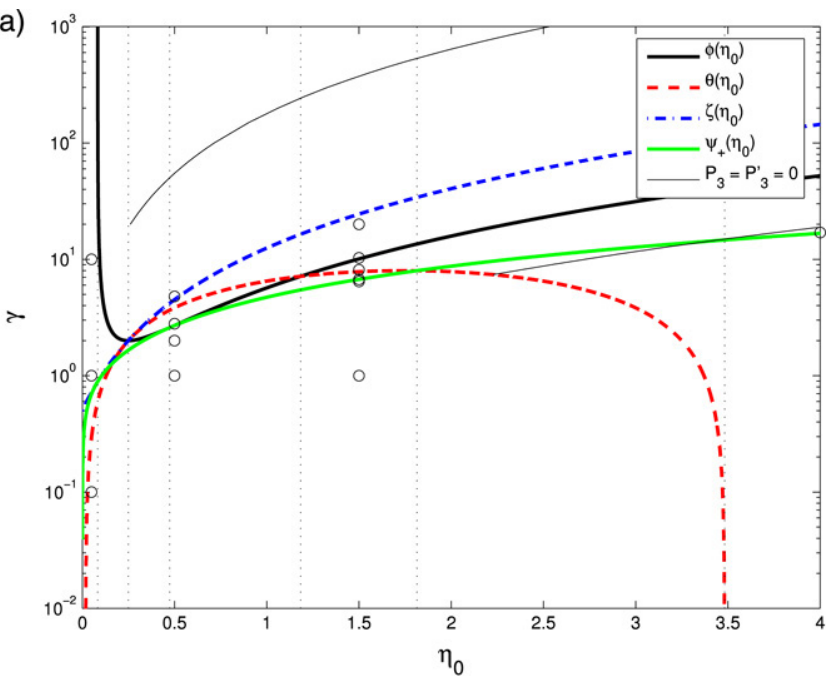

(b)

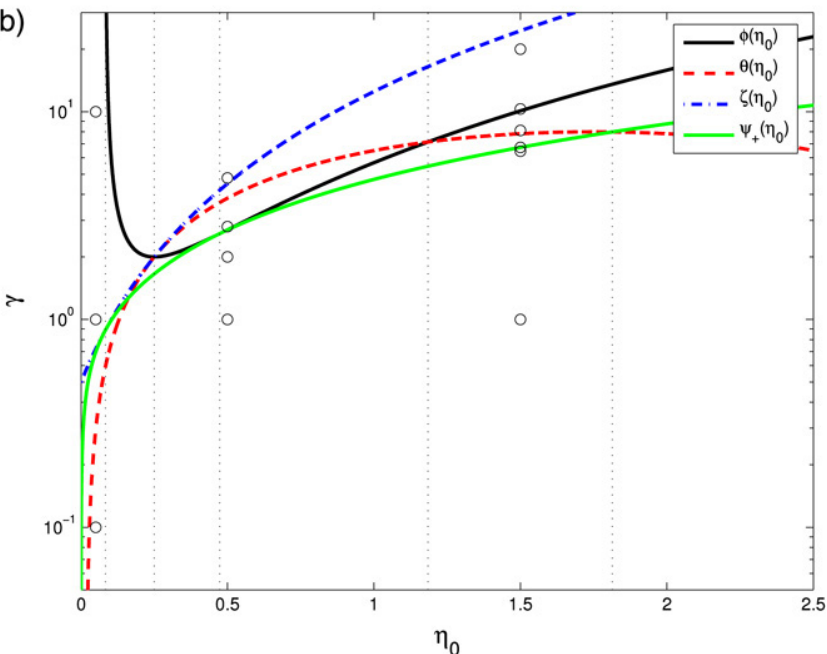

Fig. 1. The control set $\Delta$ in the case $s_{1}=+1$ and $s_{2}=+1$. (b) Displays a zoom into the region of small $\eta_{0}$. The circles represent input parameters giving the gain spectra in Figs. 2 and 3.

band (Fig. 2(b)), coalescence/splitting of gain bands (Fig. 3(a)) and coexistence of a fundamental gain band and two higher order gain bands (Fig. 3(b)). The selection of input parameters for these four cases is indicated with circles in the Fig. 1. $k_{0}$ denotes the wave number of the plane wave, while the notation $k_{\text {perturb }}$ is reserved for the wave number of the wave perturbation imposed on the plane wave background. These results are compared with numerical simulations (indicated with dots) in Figs. 2 and 3, and it is concluded that there is a fairly good agreement between the analytical and numerical approaches.

\subsection{The gain band structure in the case $s_{1}=-1, s_{2}=+1$}

In this case we can have modulational stability. We first identify regimes for which this property holds true. By carrying out the analysis based on the methodology introduced in the previous section one can, for example, prove that the plane waves are modulationally stable for the subsets of the control set where $0 \leq \eta_{0}<\frac{1}{12}, 0<\gamma<\min \left(\phi\left(\eta_{0}\right), \vartheta\left(\eta_{0}\right)\right)$ and (a)
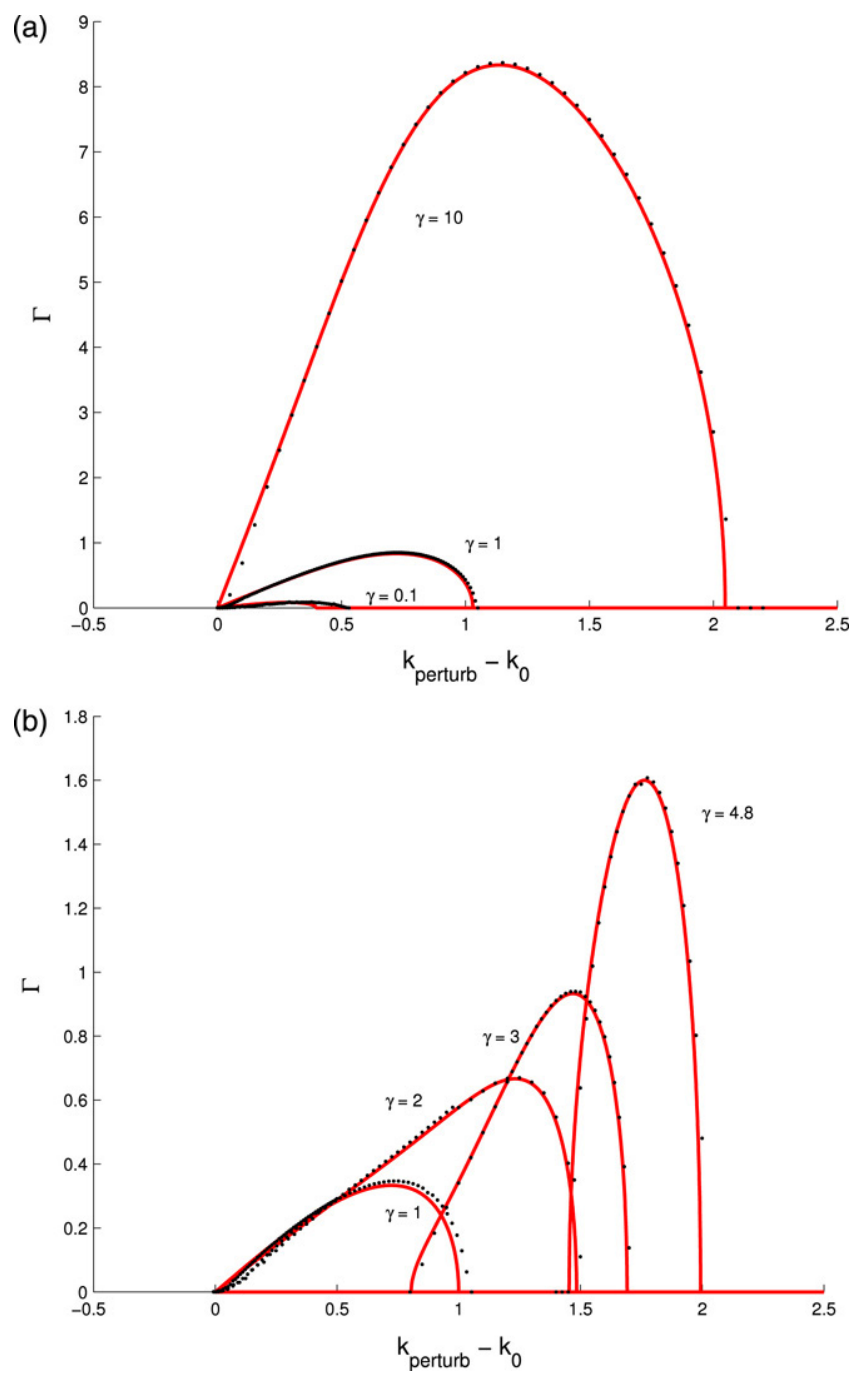

Fig. 2. Gain spectra in the case $s_{1}=+1$ and $s_{2}=+1$. (a) Existence of a single fundamental gain band in the low background wave number regime for $\left(\eta_{0}=0.05\right.$ with $\left.\gamma=0.1,1,10\right)$ and (b) conversion of a single fundamental gain to a higher order gain band ( $\eta_{0}=0.5$ with $\left.\gamma=1,2,2.8,4.8\right)$. The dots indicate the results of the numerical simulations.

$\frac{1}{12} \leq \eta_{0}<\frac{1}{4}, 0<\gamma<\max \left(\psi_{+}\left(\eta_{0}\right), \vartheta\left(\eta_{0}\right)\right)$. Here

$\phi\left(\eta_{0}\right)=-\frac{\left(4 \eta_{0}+1\right)^{3}}{24 \eta_{0}-2}$,

$\psi_{+}\left(\eta_{0}\right)=4\left(2 \eta_{0}+\sqrt{4 \eta_{0}^{2}+\eta_{0}}\right)\left(4 \eta_{0}+1\right)$,

$\vartheta\left(\eta_{0}\right)=1-16 \eta_{0}^{2}$.

Since $H_{5}(\eta=0)=b_{0}>0$ for $0 \leq \eta_{0}<\frac{1}{12}, \gamma>\phi\left(\eta_{0}\right)$, according to the previous developed theory $H_{5}$ has at least one positive zero, and hence, a fundamental gain band is excited for this regime. Notice that this is the only set of $\eta_{0}$ and $\gamma$ which produces a fundamental gain band. Even more information can be extracted by analyzing the properties of the polynomial $P_{3}$ : Since $P_{3}$ has no positive zeros in the regime $0 \leq \eta_{0}<\frac{1}{12}, \gamma>$ $\phi\left(\eta_{0}\right)$, the gain band structure only consists of the fundamental gain band in this case. Hence a typical feature of the case 

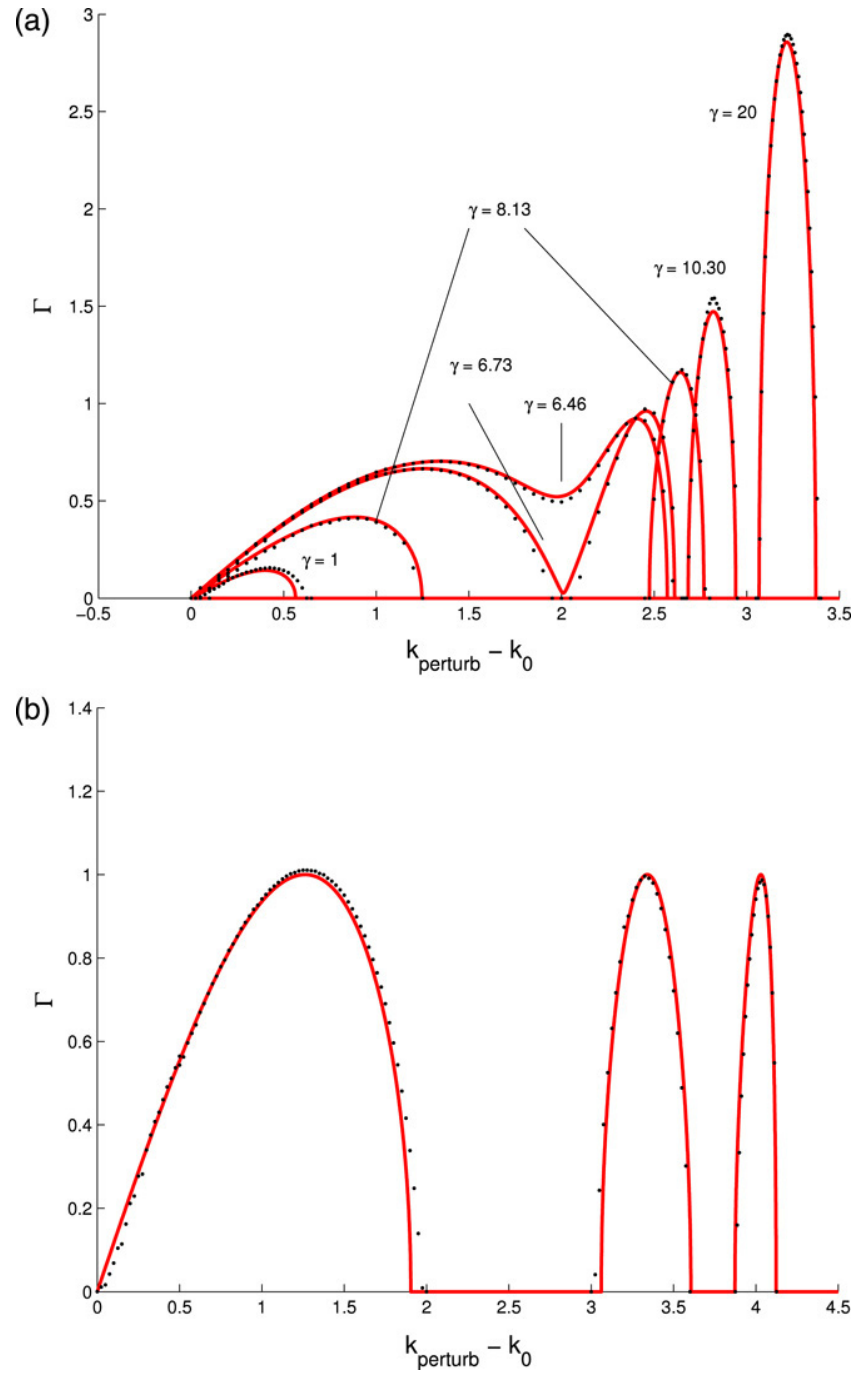

Fig. 3. Gain spectra in the case $s_{1}=+1$ and $s_{2}=+1$. (a) Splitting of a single fundamental gain into a fundamental gain band and a higher order gain band through a cusp type bifurcation, and subsequent vanishing of the fundamental gain band $\left(\eta_{0}=1.5\right.$ with $\left.\gamma=1,6.46,6.73, \gamma=8.13,10.30,20\right)$ and (b) existence of a fundamental gain band and two higher order gain bands $\left(\eta_{0}=4\right.$ with $\gamma=17)$. The dots indicate the results of the numerical simulations.

$s_{1}=-1, s_{2}=+1$, is MI in the low background wave number regime $0 \leq \eta_{0}<\frac{1}{12}$ given by a single fundamental gain band provided the effective degree of nonlocality exceeds a threshold (which depends on $\eta_{0}$ ).

One can also prove that a single higher order gain band can be formed as $\gamma$ exceeds the threshold given by the function $\psi_{+}$ for the background wave number regime $\eta_{0}>\frac{1}{4}$, due to the fact that the number of strictly positive zeros of $H_{5}$ changes from zero to two, as we pass this particular curve.

Notice also that the possibility of having coexistence of a fundamental gain band and a higher order gain band is ruled out in this case, since the maximum number of strictly positive zeros of the quintic polynomial $H_{5}$ in this case is 2 . In other words, a typical feature of this case is that the gain band structure only consists of one single band. Splitting and coalescence of gain bands do not take place.
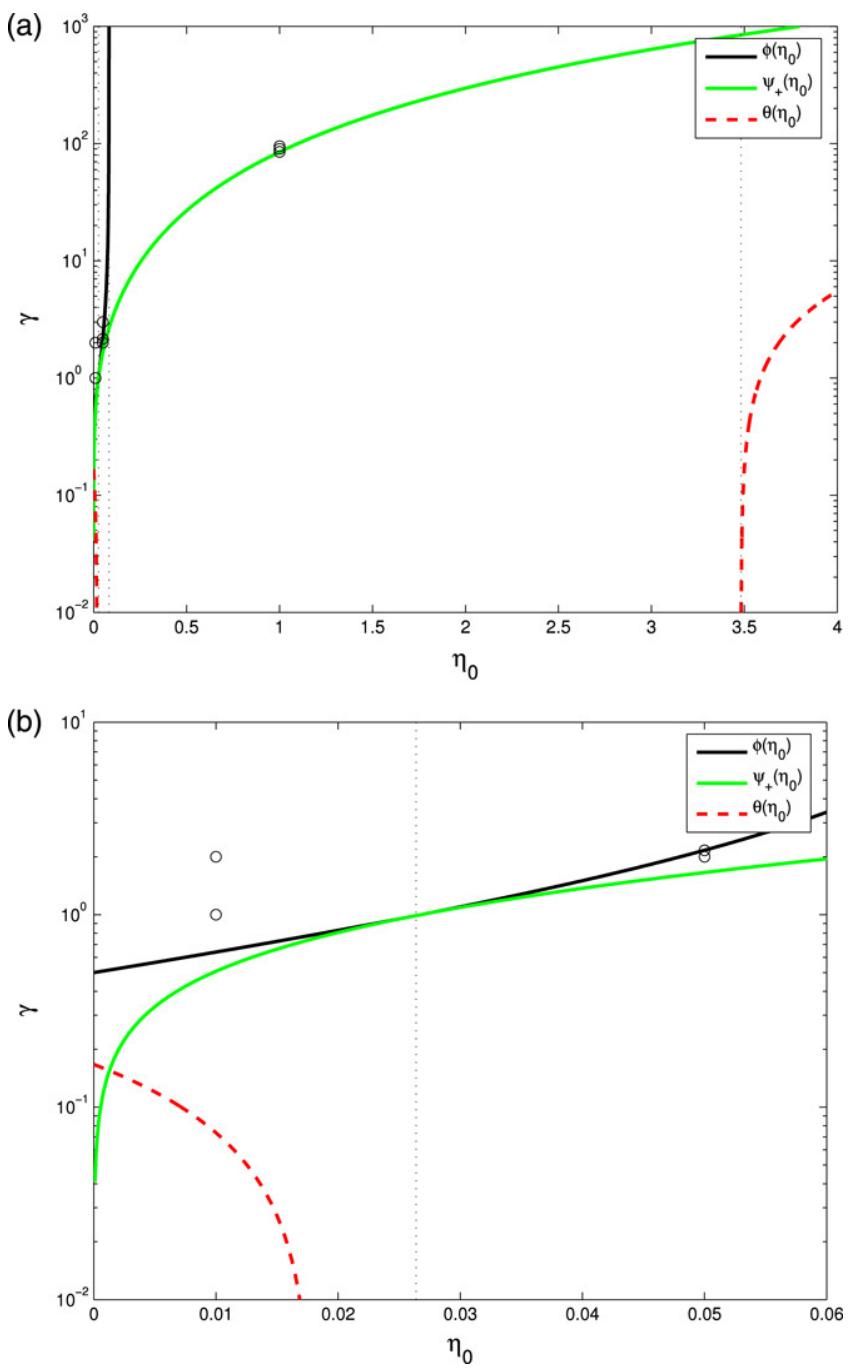

Fig. 4. The control set $\Delta$ in the case $s_{1}=-1$ and $s_{2}=+1$. (b) Displays a zoom into the region of small $\eta_{0}$. The circles represent input parameters giving the gain spectra in Fig. 5.

Again, for the purpose of identifying different gain band structures with points in the control set, we make semilogarithmic plots of the control set equipped with graphs of the functions defined in (59) as well as the function

$\theta\left(\eta_{0}\right)=\frac{8}{3} \eta_{0}^{2}-\frac{28}{3} \eta_{0}+\frac{1}{6}$

Fig. 4 shows the results. It turns out that the only regime for which $\eta_{0}>\frac{7}{4}+\sqrt{3}, 0<\gamma<\theta\left(\eta_{0}\right)$, the nontransversality condition (50) for $f=P_{3}$ can be fulfilled. In this case a single higher order gain band can be formed as we move along this curve. However, this gain band will vanish as $\gamma$ exceeds the threshold value $\theta\left(\eta_{0}\right)$.

In Fig. 5 we demonstrate graphically (a) the existence of a fundamental gain band for the low background wave number in the regime of high effective degree of nonlocality, (b) the conversion of a higher order gain band to a fundamental gain band in the low background wave number regime, and (c) the existence of a single higher order gain band in the regime 

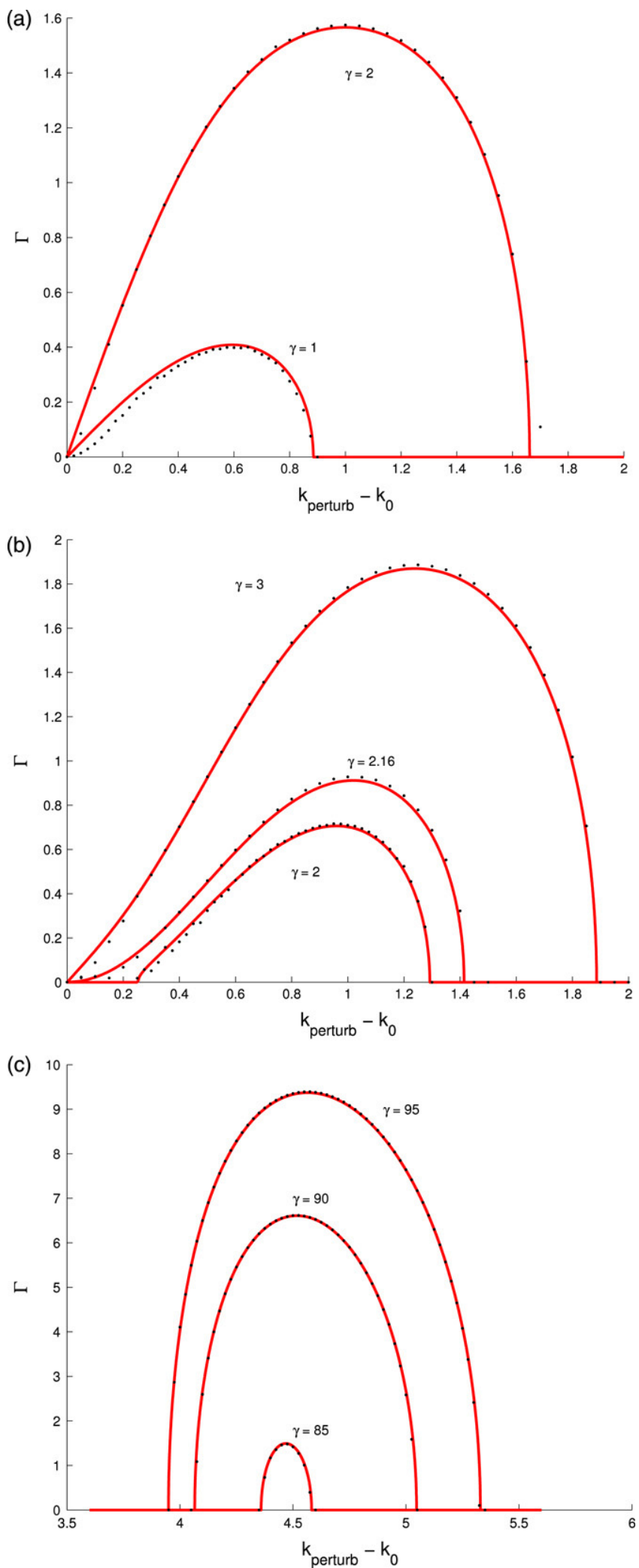

Fig. 5. Gain spectra in the case the case $s_{1}=-1$ and $s_{2}=+1$. (a) Existence of a single fundamental gain band in the low background wave number regime for the effective degree of nonlocality exceeding a certain threshold $\left(\eta_{0}=0.01\right.$ and $\gamma=1,2$ ) and (b) conversion of a higher order gain band to a fundamental gain band in the low background wave number regime $\left(\eta_{0}=0.05\right.$ and $\left.\gamma=2,2.163\right)$ and (c) existence of higher order gain band in the regime of high effective degree of nonlocality $\left(\eta_{0}=1\right.$ and $\left.\gamma=85,90,95\right)$. The dots indicate the results of the numerical simulations. of high effective degree of nonlocality. The selection of input parameters for these three cases is indicated with a ring mark in Fig. 4.

\section{Modulational instability for the oscillatory response function $\left(s_{2}=-1\right)$}

In this case we must exclude the vertical line $\eta_{0}=\frac{1}{4}$ (the resonance line) from the control set $\Delta$, since this particular line corresponds to the singular point in the spectrum $\widetilde{R}\left(2 k_{0}\right)$.

\subsection{The gain band structure in the case $s_{1}=+1, s_{2}=-1$}

In this case we can also have regimes with modulational stability. Now the analysis reveals that $Q_{2}$ has no positive zeros provided $\eta_{0}>\frac{1}{4}, \psi_{-}\left(\eta_{0}\right)<\gamma<\psi_{+}\left(\eta_{0}\right)$ where the functions $\psi_{ \pm}$originating from the factorization of $Q_{2}$ are given as

$$
\psi_{ \pm}\left(\eta_{0}\right)=4\left(4 \eta_{0}-1\right)\left(2 \eta_{0} \pm\left(\eta_{0}\left(4 \eta_{0}-1\right)\right)^{1 / 2}\right), \quad \eta_{0}>\frac{1}{4}
$$

while in the complementary set one has at least one strictly positive zero. Hence one has to search in the region $\eta_{0}>\frac{1}{4}$, $\psi_{-}\left(\eta_{0}\right)<\gamma<\psi_{+}\left(\eta_{0}\right)$ to detect parameter space producing modulational stability. By taking into account the properties of $P_{3}$ as well, one finds that the bifurcation condition $P_{3}(\eta)=$ $P_{3}^{\prime}(\eta)=0$ may take place in this regime, leading to the conclusion that excitation of a higher order gain band takes place for this particular choice of control parameters $\eta_{0}, \gamma$. We do not pursue this problem in any detail here. A further investigation reveals that the polynomial $Q_{2}$ has only one strictly positive zero for $\gamma>\phi\left(\eta_{0}\right)$ where

$\phi\left(\eta_{0}\right)=-\frac{\left(4 \eta_{0}-1\right)^{3}}{24 \eta_{0}+2}, \quad 0 \leq \eta_{0}<\frac{1}{4}$.

In the remaining part of the control set $Q_{2}$ always has two strictly positive zeros.

Now, since $P_{3}(\eta=0)=2 \gamma\left(1-4 \eta_{0}\right)>0$ for $0 \leq \eta_{0}<\frac{1}{4}$, $P_{3}$ has at least one strictly positive zero in this particular regime. Hence, by putting the information together we infer that for $0 \leq \eta_{0}<\frac{1}{4}, 0<\gamma<\phi\left(\eta_{0}\right)$, the quintic polynomial has at least three strictly positive zeros, from which it follows that we have a fundamental gain band for the background wave number bounded from above by the resonance line $\eta_{0}=\frac{1}{4}$ in that regime and that the fundamental gain bands in the oscillatory case $s_{1}=1, s_{2}=-1$ always coexist with at least one higher order gain band.

Next, when taking the monotonicity properties of $P_{3}$ into account, we can predict the exact number of gain bands formed in the MI regimes in a simple analytical way in some of these regions. More precisely, we introduce the function $\theta$ defined by (53), which in this case reads

$\theta\left(\eta_{0}\right)=\frac{1}{6}\left(16 \eta_{0}^{2}+56 \eta_{0}+1\right)$.

A careful analysis shows that $P_{3}$ has a unique strictly positive (negative) zero when $0 \leq \eta_{0}<\frac{1}{4}\left(\eta_{0}>\frac{1}{4}\right)$. By exploiting 


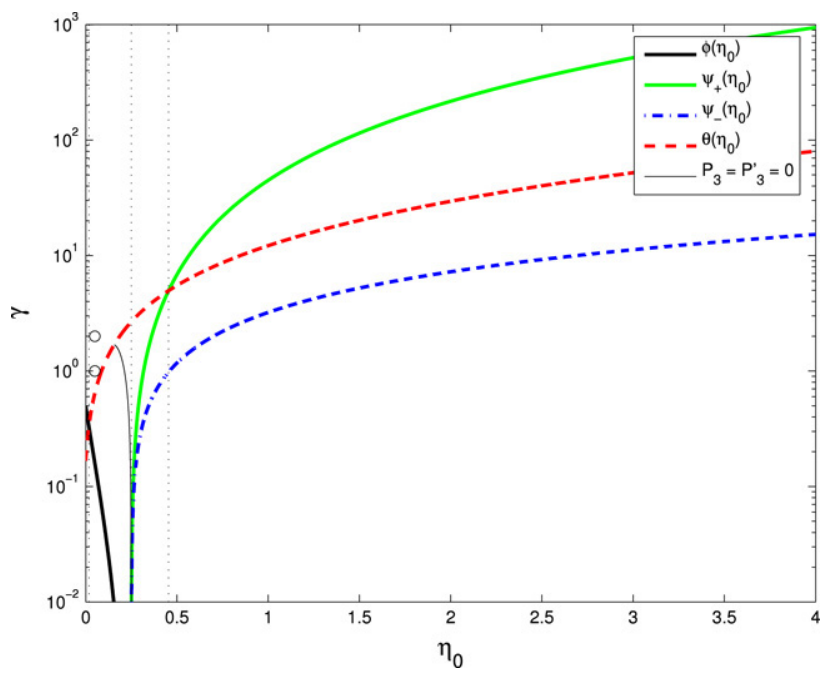

Fig. 6. The control set $\Delta$ in the case $s_{1}=+1$ and $s_{2}=-1$. The circles represent input parameters giving the gain spectra in Fig. 7.

the previously obtained information about $Q_{2}$, it is possible to show that for $0 \leq \eta_{0}<-\frac{7}{12}+\frac{1}{6} \sqrt{13}, \theta\left(\eta_{0}\right)<\gamma<\phi\left(\eta_{0}\right)$, the polynomial $H_{5}$ has exactly three positive zeros, and hence it is inferred that in this particular regime the gain band structure consists of the fundamental gain band and one single higher order gain band. For $0 \leq \eta_{0}<\frac{1}{4}, \gamma>\max \left(\phi\left(\eta_{0}\right), \theta\left(\eta_{0}\right)\right)$, one finds two positive zeros for $\mathrm{H}_{5}$, from which it follows that we have only a single gain band for this set of parameters.

In the remaining regimes the bifurcation condition (50) with $f=Q_{2}, P_{3}$ can be fulfilled, thus leading to more complex structures involving two to three gain bands and vanishing, excitation and coalescence of gain bands.

To illustrate different stability regions we first plot the curves defined by the relations (63)-(65) and (50) with $f=P_{3}$ and $s_{1}=+1, s_{2}=-1$.

Fig. 7 shows one example on the gain band structure in this case for $\eta_{0}=0.05$ with $\gamma=1$ and $\gamma=2$. It consists of a single higher order gain band for low background wave number. The selection of input parameters for this case is indicated by circles in the Fig. 6.

\subsection{The gain band structure in the case $s_{1}=-1, s_{2}=-1$}

We finally consider the oscillatory response case $s_{2}=-1$ when $s_{1}=-1$. In this case we find that the discriminant of the quadratic polynomial $Q_{2}$ is always positive. Moreover, by studying the signs of the coefficients $q_{0}$ and $q_{1}$ we find that $Q_{2}$ has at least one strictly positive zero, from which it follows that for the case $s_{1}=-1, s_{2}=-1$ the plane waves are modulationally unstable for all control parameters $\eta_{0}, \gamma$.

Next, we find that $P_{3}(\eta=0)=2 \gamma\left(4 \eta_{0}-1\right)>0$ for $\eta_{0}>\frac{1}{4}$, which means that in that particular regime $P_{3}$ has least one strictly positive zero. Moreover, the boundary function for existence of fundamental gain bands reads in this case

$\phi\left(\eta_{0}\right)=\frac{\left(4 \eta_{0}-1\right)^{3}}{24 \eta_{0}+2}, \quad \eta_{0}>\frac{1}{4}$

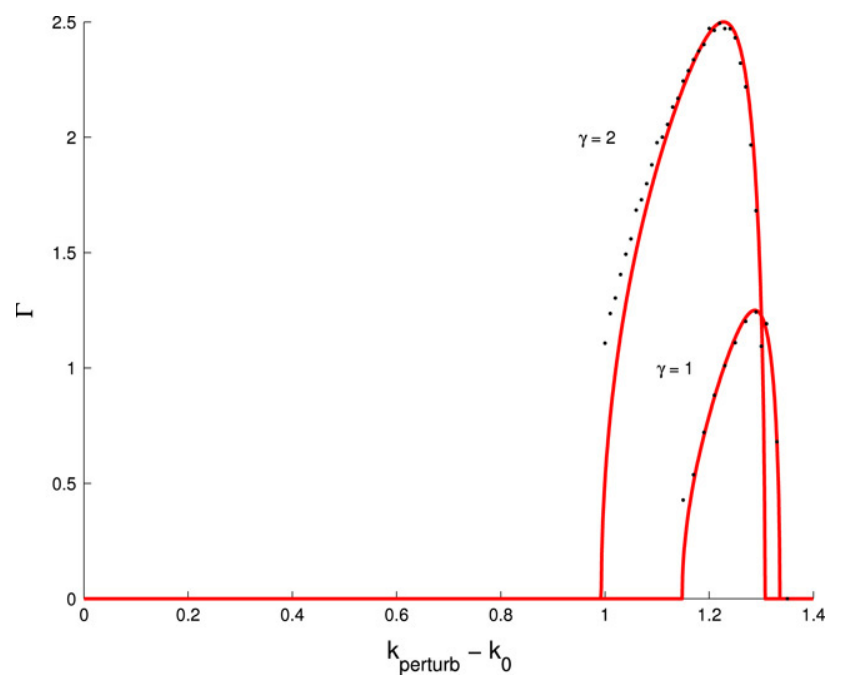

Fig. 7. Existence of a higher order gain band in the low background wave number regime in the case $s_{1}=+1$ and $s_{2}=-1\left(\eta_{0}=0.05\right.$ and $\left.\gamma=1,2\right)$. The dots indicate the results of the numerical simulations.

and one readily shows that for the regime $\eta_{0}>\frac{1}{4}, \gamma<\phi\left(\eta_{0}\right)$, $Q_{2}$ has two strictly positive zeros. Then, by adding up, the following picture emerges as a typical feature of this case: For $\eta_{0}>\frac{1}{4}, \gamma<\phi\left(\eta_{0}\right), Q_{2}$ the quintic polynomial $H_{5}$ has at least three strictly positive zeros, and hence according to the methodology described earlier, we have a fundamental gain band existing in this regime, i.e., for background wave number bounded from below by the resonance line $\eta_{0}=\frac{1}{4}$. Moreover, a typical feature in this case is the coexistence of the fundamental gain band with at least one higher order gain band. When crossing the curve $\gamma=\phi\left(\eta_{0}\right)$, the fundamental gain band vanishes due to the fact that the number of strictly positive zeros of $H_{5}$ decreases by one.

A more detailed analysis reveals that for $\eta_{0}>\frac{1}{4}, \gamma>\zeta\left(\eta_{0}\right)$, the quintic polynomial has exactly two strictly positive zeros, and hence we have only a single higher order gain band in that regime. Here

$\zeta\left(\eta_{0}\right)=\frac{1}{2}\left(4 \eta_{0}-1\right)^{2}$.

In the remaining parts of the control set the nontransversality condition (50) with $f=P_{3}$ is fulfilled, and hence we get splitting and coalescence of gain bands. One can detail the properties of the gain band structure as well as the exact number of gain bands by means of the method introduced in the present paper. We do not discuss it here in detail but rather consider some numerical examples which illustrate the MI structure.

These examples are based on the semi-logarithmic plot of the control set represented by the curves (66) and (67) as shown in Fig. 8. We select parameters $\left(\eta_{0}, \gamma\right)$ to demonstrate (a) existence of two gain bands and excitation of a higher order gain band (Fig. 9(a)) and (b) coalescence of gain bands (Fig. 9(b)). Again, a fairly good agreement between the analytical and numerical approaches is obtained. The choice of input parameters for the two cases is indicated with circles in Fig. 8. 


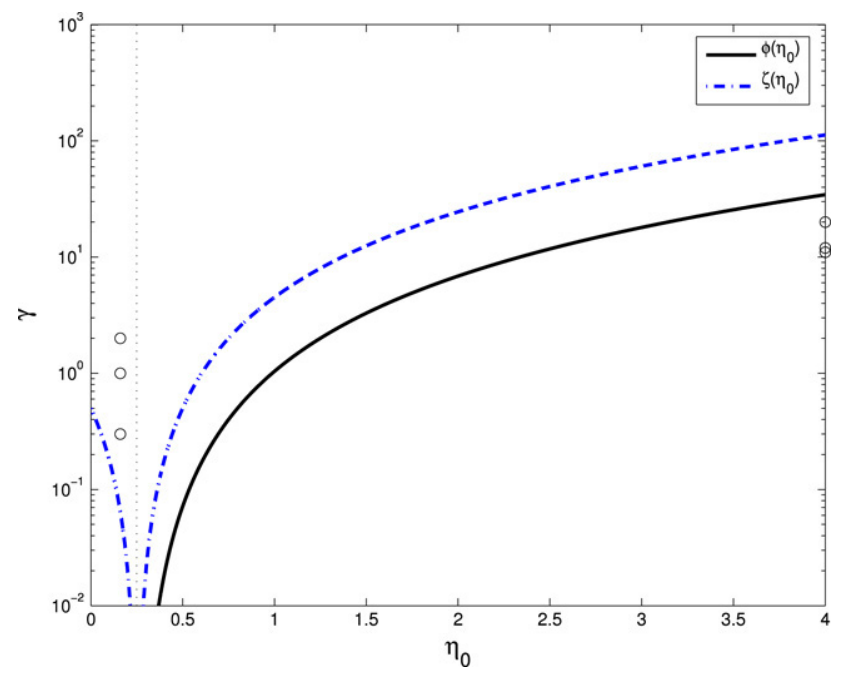

Fig. 8. The control set $\Delta$ in the case $s_{1}=-1$ and $s_{2}=-1$. The circles represent input parameters giving the gain spectra in Fig. 9.
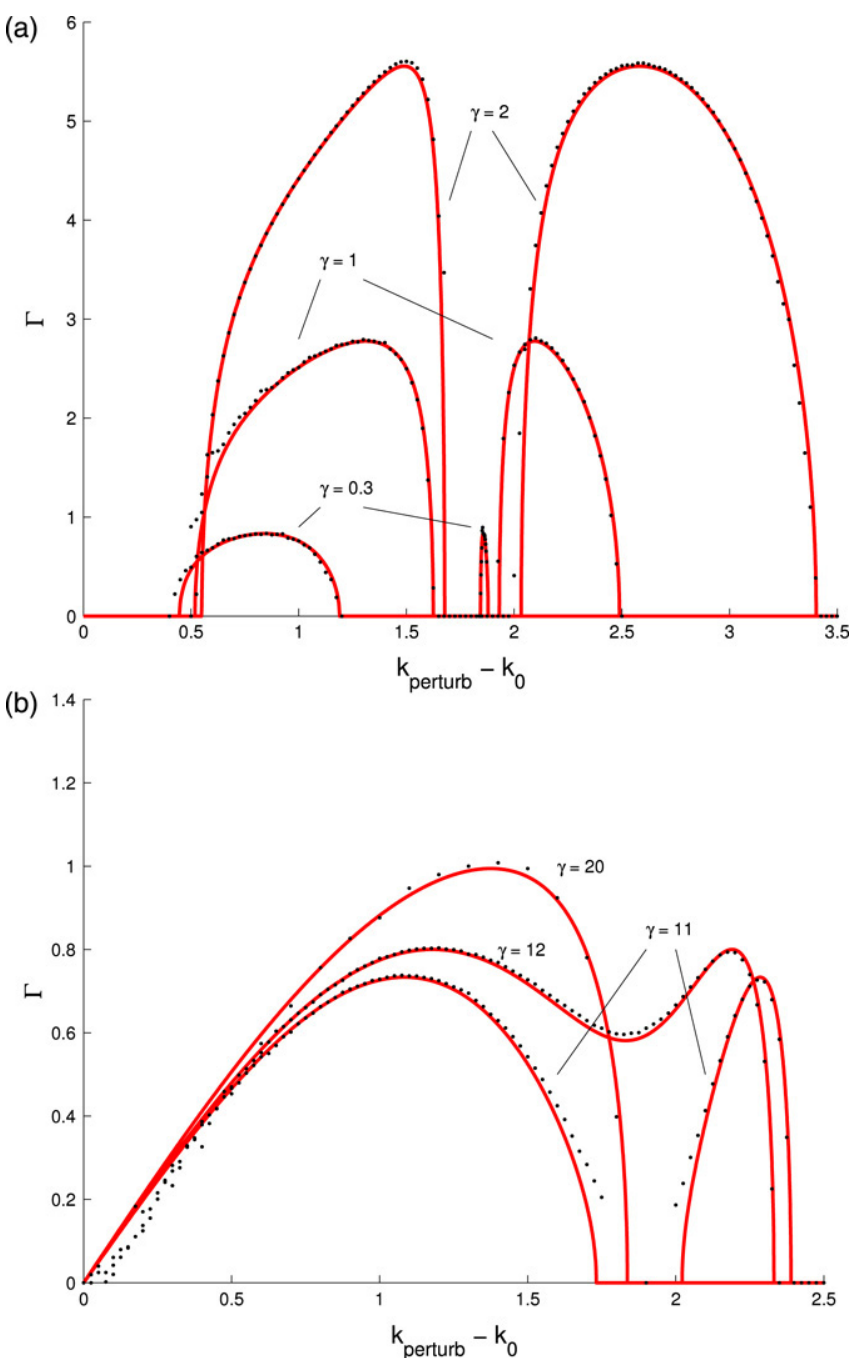

Fig. 9. Gain bands for $s_{1}=-1$ and $s_{2}=-1$. (a) Two higher order gain bands and excitation of a higher order gain band $\left(\eta_{0}=0.16\right.$ and $\left.\gamma=0.3,1,2\right)$ and (b) coalescence of gain bands ( $\eta_{0}=4$ and $\left.\gamma=11,12,20\right)$. The dots indicate the results of the numerical simulations.
6. Stability analysis of the full $\chi^{(2)}$-model versus the stability analysis of the nonlocal model

In order to investigate the role of plane waves and the MI properties of the nonlocal model (12) within the framework of the full $\chi^{(2)}$-model (1) and (2) in the non-walk-off regime $\left(r_{1}=r_{2}=0\right)$, we proceed by carrying out the same type of formal stability calculations as in [17]. The starting point is the singular perturbational problem

$$
\begin{aligned}
& \mathrm{i} \partial_{z} e_{1}+d_{1} \partial_{x}^{2} e_{1}+e_{1}^{*} e_{2}=0 \\
& \mathrm{i} \varepsilon \partial_{z} e_{2}-\beta e_{2}+d_{2} \partial_{x}^{2} e_{2}+e_{1}^{2}=0, \quad 0<\varepsilon \ll 1 .
\end{aligned}
$$

Here $\varepsilon$ is a smallness parameter which accounts for the slow variation of the amplitude $e_{2}$ in the propagation direction. In analogy with finite dimensional system theory as elaborated in for example Wasow [24] and Vasil'eva et al. [25], the nonlocal model (8)-(12) plays the role of the adiabatic approximation or the outer approximation of the perturbed problem (68) and (69). In this section we consider the validity of the stability results obtained in the previous sections by using the full $\chi^{2}$-model (68) and (69) as a starting point.

\subsection{The plane waves of the full $\chi^{(2)}$-model}

We first investigate the role of the plane wave (18) and (19) within the framework of the full model (68) and (69). We proceed as follows: By inserting the plane wave ansatz

$$
\begin{gathered}
e_{1}=\sqrt{\rho_{0}} \exp \left[\mathrm{i} \theta_{0}\right], \quad e_{2}=\widetilde{A}_{0} \exp \left[\mathrm{i} 2 \theta_{0}\right], \\
\theta_{0}=k_{0} x-\omega_{0} z
\end{gathered}
$$

where $\sqrt{\rho}_{0}$ and $\widetilde{A}_{0}$ denote the constant amplitudes of the plane waves, one ends up with the quadratic equation

$\varepsilon \omega_{0}^{2}-2\left(\alpha_{0}+\varepsilon \alpha_{1}\right) \omega_{0}+\beta_{0}=0$

as the dispersion equation for the plane waves of the $\chi^{(2)}$ model. Here the coefficients are given as

$$
\begin{aligned}
& \alpha_{0}=\frac{1}{4}\left(\beta+4 d_{2} k_{0}^{2}\right), \quad \alpha_{1}=\frac{1}{2} d_{1} k_{0}^{2}, \\
& \beta_{0}=4 \alpha_{0} \alpha_{1}-\frac{1}{2} \rho_{0} .
\end{aligned}
$$

Simple computation reveals that the discriminant of the quadratic equation (71) always is positive, provided $\varepsilon>0$. Hence plane waves exist for all choices of parameters. Notice also that the plane wave amplitudes $\sqrt{\rho}_{0}$ and $\widetilde{A}_{0}$ are coupled through the relations

$$
\begin{aligned}
& \widetilde{A}_{0}=d_{1} k_{0}^{2}-\omega_{0} \\
& \left(2 \varepsilon \omega_{0}-\beta-4 d_{2} k_{0}^{2}\right) \widetilde{A}_{0}=-\rho_{0} .
\end{aligned}
$$

The asymptotic expressions for the roots of dispersion equation (71) as $\varepsilon \rightarrow 0$ are given as

$$
\begin{aligned}
& \omega_{0}^{-}=\frac{\beta_{0}}{2 \alpha_{0}}+O(\varepsilon) \\
& \omega_{0}^{+}=\frac{2 \alpha_{0}}{\varepsilon}+O(1)
\end{aligned}
$$


provided $\alpha_{0}=O(1)$. The asymptotic expression for $\omega_{0}^{-}$is identified as the nonlocal dispersion relation (19) which we from now on will term the adiabatic branch of the dispersion relation (or the acoustic mode of the dispersion relation), while $\omega_{0}^{+}$represents the nonadiabatic branch of the dispersion relation (or the optical mode of the dispersion relation) which is a high frequency part due to the presence of the slow amplitude variation in the propagation coordinate in the $\chi^{(2)}$-model. Notice that the condition $\alpha_{0}=O(1)$ does not hold true any more in the $s_{2}=-1$ case, when the background wave number $k_{0}$ is in the vicinity of the resonance background wave number (20), i.e., $\left|k_{0}-k_{0}^{\text {res }}\right|=O(\sqrt{\varepsilon})$, which indeed reflects the case that the nonlocal model is no longer valid in this regime.

\subsection{Stability analysis of the full $\chi^{(2)}$-model}

The next problem to be addressed is the stability of the plane waves (70)-(73) within the framework of the full $\chi^{(2)}$-model.

We will focus on the following special cases

(1) The adiabatic branch of the plane waves in the limit $\varepsilon \rightarrow 0$.

(2) The nonadiabatic branch in the limit $\varepsilon \rightarrow 0$.

We proceed by perturbing the plane waves:

$e_{1}=\left[\sqrt{\rho}_{0}+A_{1}\right] \exp \left[\mathrm{i} \theta_{0}\right]$

$e_{2}=\left[\widetilde{A}_{0}+A_{2}\right] \exp \left[2 \mathrm{i} \theta_{0}\right]$

$\theta=k_{0} x-\omega_{0} z$.

Here $\sqrt{\rho}_{0}, \widetilde{A}_{0}$ and $\omega_{0}$ are given by (70), (73) and (74a) and assume the conditions $\left|A_{1}\right| \ll \sqrt{\rho}_{0}$ and $\left|A_{2}\right| \ll\left|\widetilde{A}_{0}\right|$ for the complex valued amplitudes $A_{1}$ and $A_{2}$. We obtain by linearization

$$
\begin{aligned}
& \mathrm{i} \partial_{z} A_{1}+d_{1} \partial_{x}^{2} A_{1}+2 \mathrm{i} d_{1} k_{0} \partial_{x} A_{1}-2 \mathrm{i} \widetilde{A}_{0} \operatorname{Im}\left(A_{1}\right) \\
& \quad+\sqrt{\rho_{0}} A_{2}=0, \\
& \quad \mathrm{i} \varepsilon \partial_{z} A_{2}+d_{2} \partial_{x}^{2} A_{2}+4 \mathrm{i} d_{1} k_{0} \partial_{x} A_{2}+\left(2 \varepsilon \omega_{0}^{-}-\beta-4 d_{2} k_{0}^{2}\right) A_{2} \\
& \quad+2 \sqrt{\rho_{0}} A_{1}=0 .
\end{aligned}
$$

Then, by referring the wave motion to the coordinate system (24a), one derives the system of amplitude equations of the form

$\mathrm{i} \partial_{\tau} a_{1}+d_{1} \partial_{\xi}^{2} a_{1}-2 \mathrm{i} \widetilde{A}_{0} \operatorname{Im}\left(a_{1}\right)+\sqrt{\rho_{0}} a_{2}=0$,

$\mathrm{i} \varepsilon \partial_{\tau} a_{2}+d_{2} \partial_{\xi}^{2} a_{2}+\mathrm{i} \delta \partial_{\xi} a_{2}+\mu a_{2}+2 \sqrt{\rho_{0}} a_{1}=0$,

where $a_{1}$ and $a_{2}$ denote the amplitude modulations in the new coordinate system (24a). Here $\mu$ and $\delta$ are defined as

$\mu=2 \varepsilon \omega_{0}-\beta-4 d_{2} k_{0}^{2}$

$\delta=2 k_{0}\left(2 d_{2}-\varepsilon d_{1}\right)$.

Now, when decomposing the system (76) into real and imaginary parts by assuming $a_{1}=u_{1}+\mathrm{i} v_{1}$ and $a_{2}=u_{2}+\mathrm{i} v_{2}$, one gets the set of four coupled equations

$$
\begin{aligned}
& \partial_{\tau} u_{1}+d_{1} \partial_{\xi}^{2} v_{1}-2 \widetilde{A}_{0} v_{1}+\sqrt{\rho_{0}} v_{2}=0 \\
& \partial_{\tau} v_{1}-d_{1} \partial_{\xi}^{2} u_{1}-\sqrt{\rho_{0}} u_{2}=0
\end{aligned}
$$

$\varepsilon \partial_{\tau} u_{2}+d_{2} \partial_{\xi}^{2} v_{2}+\delta \partial_{\xi} u_{2}+\mu v_{2}+2 \sqrt{\rho}_{0} v_{1}=0$

$\varepsilon \partial_{\tau} v_{2}-d_{2} \partial_{\xi}^{2} u_{2}+\delta \partial_{\xi} v_{2}-\mu u_{2}-2 \sqrt{\rho_{0}} u_{1}=0$.

Finally, by Fourier analyzing the problem (78), i.e., by introducing

$\tilde{u}_{i}(k, \tau)=\int_{-\infty}^{\infty} u_{i}(\xi, \tau) \exp [\mathrm{i} k \xi+\lambda \tau] \mathrm{d} \xi$

$\tilde{v}_{i}(k, \tau)=\int_{-\infty}^{\infty} v_{i}(\xi, \tau) \exp [\mathrm{i} k \xi+\lambda \tau] \mathrm{d} \xi$

for $i=1,2$, we end up with a linear homogeneous system of equations

B $\cdot \underline{X}=\underline{0}$

where $\mathbf{B}$ and $\underline{X}$ are the $4 \times 4$-matrix and the 4-vector, respectively, given as

$\mathbf{B}=\left(\begin{array}{cccc}\lambda & -\left(d_{1} k^{2}+2 \widetilde{A}_{0}\right) & 0 & \sqrt{\rho_{0}} \\ d_{1} k^{2} & \lambda & -\sqrt{\rho_{0}} & 0 \\ 0 & 2 \sqrt{\rho_{0}} & \varepsilon \lambda+\mathrm{i} \delta k & \mu-d_{2} k^{2} \\ -2 \sqrt{\rho_{0}} & 0 & -\left(\mu-d_{2} k^{2}\right) & \varepsilon \lambda+\mathrm{i} \delta k\end{array}\right)$ $\underline{X}=\left[\tilde{u}_{1}, \tilde{v}_{1}, \tilde{u}_{2}, \tilde{v}_{2}\right]$.

The condition for having nontrivial solutions of this linear system of equations is $\operatorname{det}(\mathbf{B})=0$ which is equivalent to the quartic equation

$\varepsilon^{2} \Lambda^{4}+\mathrm{i} \varepsilon p_{3} \Lambda^{3}+p_{2} \Lambda^{2}+\mathrm{i} p_{1} \Lambda+p_{0}=0$

in $\Lambda \equiv \lambda / d_{2} \sigma^{-2}$, where the coefficients are expressed in terms of the nondimensional parameters (11) and (35) as well as the parameter $\varepsilon$ :

$$
\begin{aligned}
p_{3}= & 4 \overline{k k}_{0}(2-\varepsilon d) \\
p_{2}= & (\varepsilon d)^{2} \eta^{2}+2(\varepsilon d)^{2} \eta_{0} \eta-2 \varepsilon d \eta s_{2} \varepsilon \Omega_{0}+4 s_{2} \varepsilon \mathrm{d} r \\
& +W\left[\eta ; \eta_{0}, s_{1}, s_{2}, \gamma, \varepsilon, \Omega_{0}, d\right] \\
p_{1}= & 4 d\left[2 s_{2} s_{1} \gamma+\varepsilon d \eta^{2}+2 \varepsilon d \eta_{0} \eta-2 \varepsilon \Omega_{0}\right] \overline{k k}_{0}(2-\varepsilon d)(81 \mathrm{c}) \\
p_{0}= & d\left[\eta\left(d \eta+2 d \eta_{0}-2 s_{2} \Omega_{0}\right) W\left[\eta ; \eta_{0}, s_{1}, s_{2}, \gamma, \varepsilon, \Omega_{0}, d\right]\right. \\
& +4 s_{2} s_{1} \gamma\left(\left(s_{2} d \eta+s_{2} d \eta_{0}-\Omega_{0}\right)\right. \\
& \left.\left.\times\left(2 \varepsilon \Omega_{0}-1-4 s_{2} \eta_{0}-s_{2} \eta\right)+s_{2} s_{1} \gamma d\right)\right] .
\end{aligned}
$$

Here

$W\left[\eta ; \eta_{0}, s_{1}, s_{2}, \gamma, \varepsilon, \Omega_{0}, d\right] \equiv \eta^{2}+\left(\left(-8-4 \varepsilon^{2} d^{2}+16 \varepsilon d\right) \eta_{0}\right.$

$$
\left.-4 \varepsilon \Omega_{0} s_{2}+2 s_{2}\right) \eta+\left(4 \eta_{0}-2 s_{2} \varepsilon \Omega_{0}+s_{2}\right)^{2} \text {. }
$$

Moreover, we have introduced the effective diffraction or dispersion coefficient $d$ defined by $d \equiv \frac{d_{1}}{d_{2}}$ and the normalized background wave frequency $\Omega_{0}$ defined by $\Omega_{0} \equiv \frac{\omega_{0}}{\beta}$. Notice that the dispersion equation (71) reads

$$
\begin{aligned}
\varepsilon & \Omega_{0}^{2}-2\left[\frac{1}{4}\left(1+4 s_{2} \eta_{0}\right)+\frac{1}{2} \varepsilon s_{2} d \eta_{0}\right] \Omega_{0}+\frac{1}{2} d s_{2} \eta_{0}\left(1+4 s_{2} \eta_{0}\right) \\
& -\frac{1}{2} s_{1} s_{2} \gamma d=0
\end{aligned}
$$


in the nondimensional quantities. Due to the fact that the discriminant of this equation is positive for $\varepsilon>0$, it possesses two real solutions which correspond to the dispersion relations of the two branches of plane waves. The system (80)-(82) constitutes the basis for our further analysis.

\subsubsection{The stability of the adiabatic branch}

Using the nondimensional form of the plane wave, the adiabatic branch (74a) is represented by the nonlocal dispersion relation (19) in the form

$\Omega_{0}^{-}=d s_{2} \eta_{0}-\frac{s_{2} s_{1} \gamma d}{1+4 s_{2} \eta_{0}}+O(\varepsilon)$

as $\varepsilon \rightarrow 0$. The leading order contributions to the coefficients $p_{0}, p_{1}, p_{2}$ and $p_{3}$ are in this case given as

$p_{3}=8 \overline{k k}_{0}+O(\varepsilon)$

$p_{2}=\eta^{2}+\left(-8 \eta_{0}+2 s_{2}\right) \eta+\left(4 \eta_{0}+s_{2}\right)^{2}+O(\varepsilon)$

$p_{1}=16 d s_{2} s_{1} \gamma \overline{k k}_{0}+O(\varepsilon)$

$p_{0}=d^{2} \eta\left[p_{03} \eta^{3}+p_{02} \eta^{2}+p_{01} \eta+p_{00}\right] /\left[1+4 s_{2} \eta_{0}\right]+O(\varepsilon)$

when employing the definition (35). Here the coefficients in (84d) are given as

$$
\begin{aligned}
& p_{03}=1+4 s_{2} \eta_{0} \\
& p_{02}=2 s_{2}-32 s_{2} \eta_{0}^{2}+2 s_{1} \gamma \\
& p_{01}=-32 s_{1} \gamma \eta_{0}+48 \eta_{0}^{2}+64 s_{2} \eta_{0}^{3}+1+12 s_{2} \eta_{0} \\
& p_{00}=-4 s_{2} \gamma^{2}-32 s_{1} \gamma \eta_{0}^{2}-16 s_{2} s_{1} \gamma \eta_{0}-2 s_{1} \gamma .
\end{aligned}
$$

We then search for asymptotic approximations for the roots $\Lambda$ of $(80)$ as $\varepsilon \rightarrow 0$. The method of undetermined gauges (i.e. balancing arguments) as for example elaborated in [28] is applicable and shows that the roots fall into two categories. The first category can be represented as a regular asymptotic expansion

$\Lambda=\Lambda_{0}+\varepsilon \Lambda_{1}+\cdots$

while the second one is given as a singular asymptotic expansion

$\Lambda=\frac{\tilde{\Lambda}_{0}}{\varepsilon}+\tilde{\Lambda}_{1}+\cdots$.

By inserting these two expansions into the quartic equation and only retaining the leading order contributions, we find that $\Lambda_{0}$ and $\widetilde{\Lambda}_{0}$ obey the quadratic equations

$p_{2} \Lambda_{0}^{2}+\mathrm{i} p_{1} \Lambda_{0}+p_{0}=0$

provided $p_{2} \neq 0$, and

$\widetilde{\Lambda}_{0}^{2}+\mathrm{i} p_{3} \widetilde{\Lambda}_{0}+p_{2}=0$.

Now, tedious algebraic computations reveal that (87) is equivalent to the dispersion relation (41) through the definitions (11), (35), (43), (47), (44a), (44b) and (84b)-(84d). Moreover, from (35), (84a) and (84b), one easily finds that

$p_{3}^{2}+4 p_{2}=4\left(\eta+4 \eta_{0}+s_{2}\right)^{2}$

from which it follows that the roots $\widetilde{\Lambda}_{0}$ are purely imaginary.

The present singular perturbational analysis clearly reveals the following typical features: When considering the stability of the nonlocal plane wave (18) using the present approach one readily sees that two timescales are involved in the wave evolution process: A slow scale for which the wave evolution is described by the nonlocal model as an adiabatic approximation to the full $\chi^{(2)}$-model given by (87), and a rapid scale describing oscillations due to the nonadiabatic part of the eigenvalues (88). The stability results of the full $\chi^{(2)}$-model thus continuously deform to the stability results of the nonlocal model (12) as $\varepsilon \rightarrow 0$ when considering the adiabatic branch of the plane waves.

\subsubsection{The nonadiabatic branch}

In this case the plane waves (70)-(73) are represented by the root $\Omega_{0}^{+}$of (82). As $\varepsilon \rightarrow 0$, this root has the asymptotic expansion

$\Omega_{0}^{+}=\frac{1+4 s_{2} \eta_{0}}{2 \varepsilon}+O(1)$

We proceed further by estimating the leading order contributions to the coefficients $p_{0}, p_{1}, p_{2}$ and $p_{3}$ :

$p_{3}=8 \overline{k k}_{0}+O(\varepsilon)$

$p_{2}=\eta^{2}-16 \eta_{0} \eta+O(\varepsilon)$

$p_{1}=8 d \overline{k k}_{0}\left(2 s_{2} s_{1} \gamma-s_{2} \eta-4 \eta_{0} \eta\right)+O(\varepsilon)$

$p_{0}=\varepsilon^{-1} q+O(1)$,

$q \equiv \eta d\left(1+4 s_{2} \eta_{0}\right)\left(-s_{2} \eta+16 s_{2} \eta_{0}+2 s_{1} \gamma\right)$

and hence the eigenvalue equation (80) assumes the nonsymmetric form

$\varepsilon^{3} \Lambda^{4}+\mathrm{i} \varepsilon^{2} p_{3} \Lambda^{3}+\varepsilon p_{2} \Lambda^{2}+\mathrm{i} \varepsilon p_{1} \Lambda+q=0$.

Again, by appealing to the method of undetermined gauges [28], one proves that the roots can be classified in terms of the asymptotic expansions

$\Lambda=\frac{\Lambda_{0}}{\varepsilon}+\Lambda_{1}+\cdots$

and

$\Lambda=\varepsilon^{-1 / 2}\left(\widetilde{\Lambda}_{0}+\varepsilon^{1 / 2} \widetilde{\Lambda}_{1}+\cdots\right)$.

The leading order contribution of (91) in the case (92) obeys

$\Lambda_{0}^{2}+\mathrm{i} p_{3} \Lambda_{0}+p_{2}=0$

and one readily shows that $p_{3}^{2}+4 p_{2}=4 \eta^{2}$, from which it follows that the roots are purely imaginary. For (93), one finds that

$\widetilde{\Lambda}_{0}^{2}=-\frac{q}{p_{2}}$ 
and by means of the asymptotic expressions (90c) and (90d), it can be inferred that for all choices of input parameters $s_{1}, s_{2}, \eta_{0}$ and $\gamma$, there is an $\eta$-range for which $q / p_{2}<0$, from which it follows that the nonadiabatic branch of the plane waves always will be modulationally unstable in the asymptotic limit $\varepsilon \rightarrow 0$.

Thus, we conclude from a physical point of view that in the parameter regimes with $s_{1}=-s_{2}$ the results from the nonlocal model, predicting modulational stability, contradict the results of the full $\chi^{(2)}$ model when accounting for both of the plane wave branches.

\section{Conclusion}

In the present paper we have focused on the modulational instability (MI) problem for the nonlocal model (8)-(12) for $\chi^{(2)}$-media. We have also compared these MI results with the predictions of the full $\chi^{(2)}$-model (1) and (2).

Our results can be summarized as follows: The plane wave solutions of the nonlocal model (12) are constructed, and a linear stability analysis of these waves is carried out. The gain spectrum of the modulational instability turns out to depend on the sign parameters $s_{1}\left(\equiv \operatorname{sign}\left(\beta d_{1}\right)\right), s_{2}\left(\equiv \operatorname{sign}\left(\beta d_{2}\right)\right)$, the effective degree of nonlocality $\gamma\left(\equiv \frac{\rho_{0} \sigma^{4}}{\left|d_{1} d_{2}\right|}\right)$ and the squared normalized background wave number $\eta_{0}\left(\equiv \sigma^{2} k_{0}^{2}\right)$. The key point in this analysis is to describe the MI in terms of a structural function in a way analogous to [3] for the MI in the conventional nonlocal NLS model. The structural function in the present case is a rational function of $\eta \equiv\left(\sigma^{2} k^{2}\right)$, where $k$ is the modulational wave number of the wave perturbation imposed on the background plane wave. The coefficients of this function depend on the parameters $\left(\eta_{0}, \gamma\right)$. The numerator of this function contains a quintic polynomial, i.e., a product of a quadratic polynomial and a cubic polynomial, and in analogy with the procedure developed in [3] the MI analysis consists of determining the number of strictly positive zeros of this polynomial as a function of the parameters $\eta_{0}$ and $\gamma$. The analysis of the MI in the nonlocal model reveals the following typical features:

- The MI is of the finite band width type. We show that the number of positive zeros of the structural function determines the gain band structure.

- For the case of exponentially decaying response function $\left(s_{2}=+1\right)$, the plane waves are always modulationally unstable for $s_{1}=+1$, while $s_{1}=-1$ is a sufficient condition for $\mathrm{MI}$ in the case of oscillatory response function $\left(s_{2}=-1\right)$.

- In both the cases $s_{2}=+1$ and $s_{2}=-1$ it is possible to separate regions in the $\left(\eta_{0}, \gamma\right)$ space producing a fundamental gain band from those where such gain bands do not exist. A prominent feature of the MI for the case $s_{2}=-1$ is that a fundamental gain band must always coexist with at least one higher order gain band.

- One can identify curves in the parameter space for which the fundamental gain band vanishes or is converted to a higher order gain band.
- The process of vanishing of the higher order gain band and coalescence of gain bands is related to the breakdown of the transversality condition at the positive zeros of the structural function, i.e., merging of positive zeros and formation of complex zeros of the structural function. This process takes place on certain curves in the $\left(\eta_{0}, \gamma\right)$ parameter space.

- The inclusion of walk-off effects in the $\chi^{(2)}$-model has no influence on wave phenomena like MI, existence of stationary soliton solutions and noncollapse, when assuming slow variation of the second harmonic amplitude with respect to the propagation coordinate $z$.

Finally, we have investigated the relationship between the MI problem for the full $\chi^{(2)}$ model and the corresponding results for the nonlocal $\chi^{(2)}$ problem. This investigation has been carried out by means of a singular perturbational approach, i.e., a smallness parameter termed $\varepsilon$ measuring the slow variation of the second harmonic amplitude with respect to the propagation coordinate has been introduced as the perturbational parameter. The results can be summarized as follows:

- We have proved that the perturbed problem (68) and (69) permits two branches of plane waves. One of these branches deforms to the plane wave of the nonlocal problem as $\varepsilon \rightarrow 0$. Moreover, it is shown that the stability results of the full model continuously deform to the MI results derived for the nonlocal model as the perturbational parameter tends to zero.

- The other plane wave branch which is singular in $\varepsilon$ and which is absent in the nonlocal $\chi^{(2)}$-model is always unstable as $\varepsilon \rightarrow 0$. Hence from a physical point of view, the nonlocal model yields an inadequate description in situations where it predicts modulational stability.

The present study indicates that the solution of the initial value problem of the full $\chi^{(2)}$ model (1) and (2) does not necessarily deform to a solution of the initial value problem of the nonlocal approximation (8)-(12). The reason for this behavior is the absence of the nonadiabatic (optical) mode in the nonlocal approximation. We conjecture that the initial value problem of the exponentially decaying case $\left(s_{2}=+1\right)$ is locally a well posed problem (which can be extended to a global result because the Sobolev norm of the solution is bounded from above) and a solution of the full $\chi^{(2)}$ model deforms to the nonlocal approximation (8)-(12) under the approximation (7), provided the nonadiabatic part is suppressed. We also believe that the relationship between the initial value problem of the full $\chi^{(2)}$ model and the initial value problem of the nonlocal approximation becomes a more complicated issue in the oscillatory case $\left(s_{2}=-1\right)$. The reason for this is the presence of the singularities in the Fourier space (=resonances). Even though we suppress the nonadiabatic, optical mode in the full $\chi^{(2)}$ model in this case it might happen that we do not get the same type of deformation of the solutions as in the exponentially decaying case. The reason is simply because the initial value problem for (8)-(12) may be mathematically illposed. 
Table A.1

The number of strictly positive zeros of $Q_{2}$

\begin{tabular}{ll}
\hline$Q_{2}(\eta)=\eta^{2}+q_{1} \eta+q_{0}$ & Number of positive zeros \\
\hline$D_{2}<0$ & 0 \\
$D_{2}>0, q_{0}>0, q_{1}>0$ & 0 \\
$D_{2}>0, q_{0}>0$ & 1 \\
$D_{2}>0, q_{0}>0, q_{1}<0$ & 2
\end{tabular}

Here we have made use of the discriminant $D_{2} \equiv q_{1}^{2}-4 q_{0}$ of $Q_{2}$.

Table A.2

The number of strictly positive zeros of $P_{3}$

\begin{tabular}{ll}
\hline$P_{3}(\eta)=-\eta^{3}+p_{2} \eta^{2}+p_{1} \eta+p_{0}$ & $\begin{array}{l}\text { Number of } \\
\text { positive zeros }\end{array}$ \\
\hline$D_{3}<0, p_{0}<0$ & 0 \\
$D_{3}>0, p_{0}<0, p_{1}>0, P_{3}\left(z_{+}\right)<0$ & 0 \\
$D_{3}>0, p_{0}<0, p_{1}<0, p_{2}>0, P_{3}\left(z_{+}\right)<0$ & 0 \\
$D_{3}>0, p_{0}<0, p_{1}<0, p_{2}<0$ & 0 \\
$D_{3}<0, p_{0}>0$ & 1 \\
$D_{3}>0, p_{0}>0, p_{1}>0$ & 1 \\
$D_{3}>0, p_{0}>0, p_{1}<0, p_{2}<0$ & 1 \\
$D_{3}>0, p_{0}>0, p_{1}<0, p_{2}>0, P_{3}\left(z_{ \pm}\right)<0$ & 1 \\
$D_{3}>0, p_{0}>0, p_{1}<0, p_{2}>0, P_{3}\left(z_{ \pm}\right)>0$ & 1 \\
$D_{3}>0, p_{0}<0, p_{1}>0, P_{3}(z+)>0$ & 2 \\
$D_{3}>0, p_{0}<0, p_{1}<0, p_{2}>0, P_{3}\left(z_{-}\right)<0<P_{3}\left(z_{+}\right)$ & 2 \\
$D_{3}>0, p_{0}>0, p_{1}<0, p_{2}>0, P_{3}\left(z_{-}\right)<0<P_{3}\left(z_{+}\right)$ & 3
\end{tabular}

Here we have made use of the discriminant $D_{3} \equiv p_{2}^{2}+3 p_{1}$ of the derivative of $P_{3}$ and the extremal points $z_{ \pm} \equiv \frac{p_{2} \pm \sqrt{D_{3}}}{3}\left(D_{3} \geq 0\right)$.

\section{Acknowledgements}

This research is supported by the Danish Technical Research Council (Grant No. 26-00-0355), the Australian Research Council, and the Australian Photonics Cooperative Research Centre. J. Wyller acknowledges support from The Research Council of Norway under Grant No. 153405/432. The present work was started in 2003/04 when J. Wyller was a Visiting Fellow at Australian National University (ANU). The authors would like to thank Yuri Kivshar and Dragomir Neshev (ANU), Arkadi Ponossov, Gaute Einevoll and Patrick Blomquist (Norwegian University of Life Sciences) for many fruitful and stimulating discussions during the preparation phase of the present paper. The authors would also like to thank the reviewers for constructive remarks.

\section{Appendix. The counting principle for positive zeros}

Let us assume that quintic polynomial $H_{5}$ can be factorized into a product of a cubic polynomial $P_{3}$ and a quadratic polynomial $Q_{2}$ :

$$
\begin{aligned}
& P_{3}(\eta)=-\eta^{3}+p_{2} \eta^{2}+p_{1} \eta+p_{0} \\
& Q_{2}(\eta)=\eta^{2}+q_{1} \eta+q_{0}
\end{aligned}
$$

where the coefficients are continuous functions almost everywhere of the control parameter set $\Delta$ contained in $\mathbf{R}^{n}$. Tables A.1 and A.2 conveniently summarize the number of strictly positive zeros of $Q_{2}$ and $P_{3}$ as functions of their respective coefficients in the case of transversal crossings, i.e. when $f(\eta)=0, f^{\prime}(\eta) \neq 0, f=P_{3}, Q_{2}$. The constraints put on the coefficients of the polynomials $Q_{2}$ and $P_{3}$ in these tables clearly divide the control set $\Delta$ into a union of disjoint, open subsets $\Delta_{i}^{f}$, where the number of positive zeros of the respective polynomial is constant on each subset $\Delta_{i}^{f}$. This enables us to formulate the counting principle for the number of strictly positive zeros of $\mathrm{H}_{5}$ in the case of transversal crossings: Let $\Delta_{i}^{Q_{2}}$ be an open subset of the control set $\Delta$ for which $Q_{2}$ has $N_{i}\left(Q_{2}\right)$ strictly positive zeros $\left(N_{i}\left(Q_{2}\right)=0,1\right.$ or 2$)$ and $\Delta_{j}^{P_{3}}$ be a subset of the control set for which $P_{3}$ has $N_{j}\left(P_{3}\right)$ strictly positive zeros $\left(N_{j}\left(P_{3}\right)=0,1,2\right.$ or 3$)$. Assume that the intersection $\Delta_{i}^{Q_{2}} \cap \Delta_{j}^{P_{3}}$ is nonempty. Then the number $\mathrm{N}_{i, j}\left(\mathrm{H}_{5}\right)$ of strictly positive zeros of $\mathrm{H}_{5}$ on the intersection $\Delta_{i}^{Q_{2}} \cap \Delta_{j}^{P_{3}}$ is given as $N_{i, j}\left(H_{5}\right)=N_{i}\left(Q_{2}\right)+N_{j}\left(P_{3}\right)$.

The conditions $H_{0}(\eta=0)=0$ and $H_{5}(\eta)=H_{5}^{\prime}(\eta)=0$ yield analytical descriptions of the boundary of the nonempty intersection $\Delta_{i}^{Q_{2}} \cap \Delta_{j}^{P_{3}}$ in the control set $\Delta$. When crossing such boundaries, the number of positive zeros of $H_{0}$ changes.

In our case the set of coefficients is given as

$$
\begin{aligned}
& p_{2}=2\left(4 \eta_{0}-s_{2}\right) \\
& p_{1}=-\left(4 \eta_{0}+s_{2}\right)^{2}+2 s_{2} s_{1} \gamma \\
& p_{0}=2 s_{2} s_{1} \gamma\left(4 \eta_{0}+s_{2}\right) \\
& q_{1}=-2 \frac{16 \eta_{0}^{2}-1-s_{2} s_{1} \gamma}{4 \eta_{0}+s_{2}} \\
& q_{0}=\left(4 \eta_{0}+s_{2}\right)^{2}-2 s_{1} s_{2} \gamma \frac{12 \eta_{0}-s_{2}}{4 \eta_{0}+s_{2}} \\
& f_{1}=-2\left(4 \eta_{0}-s_{2}\right) \\
& f_{0}=\left(4 \eta_{0}+s_{2}\right)^{2} .
\end{aligned}
$$

This means that the control set is two dimensional for a given set of sign parameters $\left(s_{1}, s_{2}\right)=( \pm 1, \pm 1)$. Hence, in order to predict the gain band structure for a given set of input parameters $\left(\eta_{0}, \gamma\right)$, one computes the set of corresponding coefficients $\left(p_{0}, p_{1}, p_{2}, q_{0}, q_{1}\right)$ by means of the formulas (A.3) and (A.4). The number of strictly positive zeros of $Q_{2}$ and $P_{3}$ are determined by means of Tables 1 , A.1 and A.2. The counting principle then yields the total number of strictly positive zeros of $\mathrm{H}_{5}$.

\section{References}

[1] W.Z. Królikowski, O. Bang, J. Wyller, J.J. Rasmussen, Optical beams in nonlocal nonlinear media, Acta Phys. Polon. A 103 (2003) 133-147.

[2] W.Z. Królikowski, O. Bang, N.L. Nikolov, D. Neshev, J. Wyller, J.J. Rasmussen, D. Edmundsson, Modulational instability, solitons and beam propagation in spatially nonlocal nonlinear media, J. Opt. B 6 (2004) S288-S294.

[3] J. Wyller, W.Z. Królikowski, O. Bang, J.J. Rasmussen, Generic features of modulational instability in a nonlocal Kerr media, Phys. Rev. E 66 (2002) 066615.

[4] W.Z. Królikowski, O. Bang, J.J. Rasmussen, J. Wyller, Modulational instability in nonlocal nonlinear Kerr media, Phys. Rev. E 64 (2001) 016612.

[5] O. Bang, W.Z. Królikowski, J. Wyller, J.J. Rasmussen, Collapse arrest and soliton stabilization in nonlocal nonlinear media, Phys. Rev. E 66 (2002) 046619. 
[6] W.Z. Królikowski, O. Bang, Solitons in nonlocal nonlinear media: Exact solutions, Phys. Rev. E 63 (2001) 016610.

[7] D. Suter, T. Blasberg, Stabilisation of transverse solitary waves by a nonlocal response of the nonlinear medium, Phys. Rev. A 48 (1993) 4583-4587.

[8] J.P. Gordon, R.C.C. Leite, R.S. Moore, S.P.S. Porto, J.R. Whitney, Longtransient effects in lasers with inserted liquid samples, J. Appl. Phys. 36 (1965) 3.

[9] S. Akhmanov, D. Krindach, A. Migulin, A. Sukhorukov, R. Khokhlov, Thermal self-actions of laser beams, IEEE J. Quantum Electron. QE-4 (1968) 568-575.

[10] S. Gatz, J. Herrmann, Anisotropy, nonlocality, and space-charge field displacement $(2+1)$-dimensional self-trapping in biased photorefractive crystals, Opt. Lett. 23 (1998) 1176-1178.

[11] D.W. McLaughlin, D.J. Muraki, M.J. Shelley, X. Wang, A paraaxial model for optical self-focusing in nematic liquis crystals, Physica D 88 (1995) 55-81;

M. Karpierz, Solitary waves in liquid crystalline waveguides, Phys. Rev. E 66 (2002) 036603.

[12] C. Conti, M. Peccianti, G. Assanto, Route to nonlocality and observations of accesible solitons, Phys. Rev. Lett. 91 (2003) 073901.

[13] F. Dalfovo, S. Giorgini, L.P. Pitaevski, S. Stringari, Theory of Bose-Einstein condenasation in trapped gases, Rev. Modern Phys. 71 (1999) 463.

[14] N.I. Nikolov, D. Neshev, O. Bang, W.Z. Królikowski, Quadratic solitons as nonlocal solitons, Phys. Rev. E 68 (2003) 036614;

I.V. Shadrivov, A.A. Zharov, Dynamics of optical spatial solitons near the interface between two quadratically nonlinear media, J. Opt. Soc. Amer. B 19 (2002) 596-602.

[15] C.R. Menyuk, R. Schieck, L. Torner, Solitary waves due to $\chi^{(2)}: \chi^{(2)}$ cascading, J. Opt. Soc. Amer. B 11 (1994) 2434;

O. Bang, Dynamical equations for wave packets with both quadratic and cubic response, J. Opt. Soc. Amer. B 14 (1997) 51-61.

[16] H. He, P. Drummond, B.A. Malomed, Modulational instability in dispersive optical systems with cascaded nonlinearity, Opt. Commun. 123 (1996) 394-402.

[17] A.V. Buryak, P. Di Trapani, D.V. Skryabin, S. Trillo, Optical solitons due to quadratic nonlinearities: From basic physics to futuristic applications, Phys. Rep. 370 (2002) 63-235.

[18] F.Ö. Ilday, K. Beckwitt, Y.-F. Chen, H. Lim, F.W. Wise, Controllable Raman-like nonlinearities from nonstationary, cascaded processes, J. Opt. Soc. Amer. B 21 (2004) 376-383.

[19] L. Bergé, V.K. Mezentsev, J.J. Rasmussen, J. Wyller, Formation of stable solitons in quadratic nonlinear media, Phys. Rev. A 52 (1) (1995) R28-R31.

[20] S.K. Turitsyn, Stability of two-and three dimensional optical solitons in media with qaudratic nonlinearity, Pis'ma Zh. Eskp. Teor. Fiz. 61 (1995) 458-460, JETP Lett. 61 (1995) 469.

[21] D.E. Pelinovsky, A.V. Buryak, Y.S. Kivshar, Instability of solitons governed by quadratic nonlinearities, Phys. Rev. Lett. 75 (1995) 591-595.

[22] P.V. Larsen, M.P. Sørensen, O. Bang, W.Z. Królikowski, S. Trillo, Nonlocal description of $X$ waves in in quadratic nonlinear materials, Phys. Rev. E 73 (2006) 036614.

[23] F. Biancalana, D.V. Skryabin, P.St.J. Russel, Four-wave mixing instabilities in photonic-crystal and tapered fibers, Phys. Rev. E 178 (2003) 046603.

[24] W. Wasow, Asymptotic Expansions for Ordinary Differential Equations, Wiley Interscience, 1965.

[25] A.B. Vasil'eva, V.B. Butuzov, L.V. Kalachev, The Boundary Function Method for Singular Perturbation Problems, SIAM Studies in Applied Mathematics, 1995.

[26] J. Wyller, P. Blomquist, G.T. Einevoll, Turing instability and pattern formation in a two-population neuronal network model, Physica D 225 (2007) 75-93.

[27] T.B. Benjamin, J.E. Feir, The desintegration of wave trains on deep water, Part I, J. Fluid Mech. 27 (1967) 417.

[28] J.G. Simmons, J.E. Mann, A First Look at Perturbation Theory, Kreiger, Malabar, Florida, 1986. 